\title{
Identification of cell-specific targets of sumoylation during mouse spermatogenesis
}

\author{
Yuxuan Xiao, Daniel Pollack, Miriam Andrusier, Avi Levy, Myrasol Callaway ${ }^{1}$, Edward Nieves ${ }^{1}$, \\ Prabhakara Reddi ${ }^{4}$ and Margarita Vigodner ${ }^{2,3}$ \\ Department of Biology, Stern College, Yeshiva University, New York, New York, USA, ${ }^{1}$ Laboratory for Macromolecular \\ Analysis and Proteomics, Albert Einstein College of Medicine, Bronx, New York, USA, ${ }^{2}$ Department of Biology, \\ Stern College, Yeshiva University, 245 Lexington Avenue, New York, New York 10016, USA, ${ }^{3}$ Department of \\ Developmental and Molecular Biology, Albert Einstein College of Medicine, Bronx, New York, USA and \\ ${ }^{4}$ Department of Pathology, University of Virginia, Charlottesville, Virginia, USA
}

Correspondence should be addressed to M Vigodner; Email: vigodner@yu.edu

\begin{abstract}
Recent findings suggest diverse and potentially multiple roles of small ubiquitin-like modifier (SUMO) in testicular function and spermatogenesis. However, SUMO targets remain uncharacterized in the testis due to the complex multicellular nature of testicular tissue, the inability to maintain and manipulate spermatogenesis in vitro, and the technical challenges involved in identifying lowabundance endogenous SUMO targets. In this study, we performed cell-specific identification of sumoylated proteins using concentrated cell lysates prepared with de-sumoylation inhibitors from freshly purified spermatocytes and spermatids. One-hundred and twenty proteins were uniquely identified in the spermatocyte and/or spermatid fractions. The identified proteins are involved in the regulation of transcription, stress response, microRNA biogenesis, regulation of major enzymatic pathways, nuclear-cytoplasmic transport, cell-cycle control, acrosome biogenesis, and other processes. Several proteins with important roles during spermatogenesis were chosen for further characterization by co-immunoprecipitation, co-localization, and in vitro sumoylation studies. GPS-SUMO Software was used to identify consensus and non-consensus sumoylation sites within the amino acid sequences of the proteins. The analyses confirmed the cell-specific sumoylation and/or SUMO interaction of several novel, previously uncharacterized SUMO targets such as CDK1, RNAP II, CDC5, MILI, DDX4, TDP-43, and STK31. Furthermore, several proteins that were previously identified as SUMO targets in somatic cells (KAP1 and MDC1) were identified as SUMO targets in germ cells. Many of these proteins have a unique role in spermatogenesis and during meiotic progression. This research opens a novel avenue for further studies of SUMO at the level of individual targets.
\end{abstract}

Reproduction (2016) 151 149-166

\section{Introduction}

Spermatogenesis includes the proliferation of spermatogonia through mitosis, the production of round spermatids from spermatocytes by meiosis, and the post-meiotic maturation of spermatids, termed spermiogenesis. Abnormalities during any of these processes can result in the production of malfunctioning sperm, which may lead to infertility, spontaneous abortion, or birth defects. These facts emphasize the need for a better understanding of spermatogenesis and its regulation, particularly through the characterization of the molecules that have not been well studied in the testis but that regulate important pathways in other tissues.

Various protein post-translational modifications (PTMs) regulate spermatogenesis, one of which is the attachment of small ubiquitin-like modifiers (SUMOs) in a process termed sumoylation. SUMOs are structurally similar to ubiquitin. However, their amino acid sequences differ greatly, with only $\sim 18 \%$ similarity (Bayer et al. 1998). Four SUMO paralogs have been identified in mammals: SUMO1-SUMO4. SUMO1 (also named sentrin) shares $\sim 50 \%$ homology with SUMO2 and SUMO3, which are usually referred to as SUMO2/3 given that they are 95\% identical. During sumoylation, an isopeptide bond forms between a SUMO and the lysine residue of its substrate. This process requires a SUMO-activating enzyme (E1), a SUMO-conjugating enzyme (E2), and a SUMO ligase (E3) (Yeh et al. 2000, Wang \& Dasso 2009, Yeh 2009, Wilkinson \& Henley 2010). Sumoylation often occurs on a target lysine residue within the consensus sequence: $\psi-K-X-D / E$, where $\psi$ is a hydrophobic amino acid and $X$ can be any amino acid (Rodriguez et al. 2001). However, not all consensus sequences are sumoylated, and sumoylation often occurs outside of the consensus sequence (Blomster et al. 2010). Notably, SUMO2/3 but not SUMO1 contain the consensus sequence, and mixed 
SUMO chains with a terminal SUMO1 have been reported (Rodriguez et al. 2001). Sumoylation is a dynamic process that can be reversed through the activity of sentrin-specific proteases (SENPs) by the cleavage of the isopeptide bond between the SUMO moiety and the substrate (Mukhopadhyay \& Dasso 2007, Yeh 2009, Hannoun et al. 2010, Wilkinson \& Henley 2010). A diverse set of SUMO target proteins has been identified in somatic cells, including factors that regulate transcription, replication, DNA repair, RNA metabolism, translation, and cellular transport. In addition to the numerous targets of sumoylation that have been identified, there is a growing list of proteins that interact with SUMO non-covalently (Song et al. 2004, 2005, Chupreta et al. 2005, Lin et al. 2006, Kerscher 2007).

Several developmental processes, including spermatogenesis, have been studied in SUMO1-knockout mice (Zhang et al. 2008). In contrast to a previously published study that reported abnormal development of the palate in SUMO1 knockouts (Alkuraya et al. 2006), Zhang et al. found no abnormalities in mouse development, suggesting that SUMO2 and SUMO3 compensated for the functions of SUMO1. Although these results require further evaluation, they suggest that the SUMOconjugating machinery, and not an individual SUMO isoform, should be the target of future experiments aiming to inhibit sumoylation. Unfortunately, knockout mice for UBC9, a SUMO-conjugating enzyme, show early embryonic lethality and severe disruptions in mitosis, a finding that supports the indispensable role of sumoylation in mitotic progression (Nacerddine et al. 2005). We and other groups have studied SUMO1 and SUMO2/3 in mouse and human spermatogenesis using advanced cell-imaging techniques and immunodetection analyses. SUMO localized to spermatogonia, the sex chromosomes and the centromeric heterochromatin of spermatocytes, the chromocenters of round spermatids, the centrosome area of elongating spermatids, and the nuclei of testicular somatic cells. These findings are consistent with the diverse and potentially multiple roles of SUMO in testicular function and spermatogenesis, such as spermatogonia proliferation, meiotic sex chromosome inactivation, centromeric heterochromatin organization, and reshaping the spermatid nucleus (Rogers et al. 2004, Vigodner \& Morris 2005, Vigodner et al. 2006, Brown et al. 2008, Metzler-Guillemain et al. 2008, Vigodner 2009). In agreement with these results, one study (La Salle et al. 2008) showed the dynamic nature of the gene expression levels related to sumoylation during spermatogenesis. Recent studies from our group also revealed changes in global sumoylation following the induction of various stresses in germ cells and sperm (Shrivastava et al. 2010, 2014). Although these studies provided important initial information about the possible roles of sumoylation in spermatogenesis, little progress has been made in understanding how SUMO regulates the suggested functions. As has been shown in somatic cells, the identification of targets for sumoylation is a critical step toward understanding its cellular functions (Andersen et al. 2009, Golebiowski et al. 2009, Sarge \& Park-Sarge 2009, Tatham et al. 2009). TOP2A and synaptonemal complex proteins (SYCP1 and SYCP2) have been co-immunoprecipitated with SUMO from testicular lysates, as shown by our group (Shrivastava et al. 2010) and others (Brown et al. 2008) respectively. Hundreds of SUMO targets have been identified in somatic cells. However, with the exception of TOP2A and SYCPs, SUMO targets remain uncharacterized in the testis. This knowledge gap is due to the complex, multicellular nature of testicular tissue, the inability to maintain and manipulate spermatogenesis in vitro, and the technical challenges involved in identifying low-abundance endogenous SUMO targets. To overcome some of these difficulties, we recently optimized the identification of sumoylated proteins using concentrated cell lysates, isopeptidase inhibitors to prevent de-sumoylation, and a large amount of anti-SUMO antibody crosslinked to agarose beads (Xiao et al. 2015). Using this approach, we recently identified a sumoylome of human sperm (Vigodner et al. 2013). Several additional sperm targets were identified by another group (Marchiani et al. 2014). In this study, numerous sumoylated proteins were uniquely identified in the spermatocyte and/or spermatid fractions using lysates prepared from purified spermatogenic cells. Several proteins with important roles during spermatogenesis were further characterized by co-immunoprecipitation (IP), co-localization, and in vitro sumoylation studies.

\section{Materials and methods}

\section{Mice, cell lines, reagents, and antibodies}

C57BL/6NCrl mice were purchased from Charles River (Kingston, NY, USA). The Animal Committee of Albert Einstein College of Medicine, Yeshiva University approved all animal protocols. The mouse Sertoli cell line 15P-1 (ATCC, CRL-2618) and human embryonic kidney 293 (HEK-293; ATCC CRL-1573) cells were purchased from ATCC (Manassas, VA, USA) and grown in DMEM with 5\% fetal bovine serum (Life Technologies, 16140-071), 5\% bovine growth serum (Fisher Scientific, Waltham, MA, USA; SH30541.03), 1\% penicillin/streptomycin (Life Technologies, 15140-122), and $0.5 \%$ Fungizone (Life Technologies, $15290-018$ ) at $32{ }^{\circ} \mathrm{C}$ with $5 \%$ $\mathrm{CO}_{2}$. The primary human Sertoli cell line (Lonza, MM-HSE2305) was purchased from Lonza Group Ltd (Walkersville, MD, USA) and cultured in the Sertoli Cell Growth Medium Bullet Kit (Lonza, 00191053) at $37{ }^{\circ} \mathrm{C}$ with $5 \% \mathrm{CO}_{2}$.

All chemicals were purchased from Sigma-Aldrich unless otherwise noted. All reagents for western blotting and immunofluorescence were purchased from Life Technologies unless otherwise noted. The detergent-removal spin columns (87778), the screw-cap spin columns (69705), and the BCA Protein Assay Kit (23227) were purchased from Thermo 
Scientific (Rockford, IL, USA). The Whole-Cell Extraction Kit (2910) was purchased from Millipore (Temecula, CA, USA). Information regarding the source, vendor, and dilution of the antibodies used in this study is summarized in Supplementary Table 1, see section on supplementary data given at the end of this article.

\section{Germ cell separation by velocity sedimentation (STA-PUT)}

Mouse testicular germ cells were separated using a STA-PUT sedimentation velocity cell separator (ProScience, Inc., Scarborough, ON, Canada) and a published procedure (La Salle et al. 2009). The details of the procedure are summarized in the Supplementary Materials, see section on supplementary data given at the end of this article.

\section{Flow cytometry analysis}

Chosen STA-PUT fractions were centrifuged at $500 \mathrm{~g}$ for $7 \mathrm{~min}$ at $4{ }^{\circ} \mathrm{C}$ followed by aspiration of all but $1 \mathrm{ml}$ of supernatant. The pellets were then resuspended with a brief, gentle vortex. From each resulting cell suspension, $150 \mu \mathrm{l}$ was individually mixed with propidium iodide (PI) staining solution (PBS with 1\% (v/v) RNase A, $10 \mu \mathrm{g} / \mathrm{ml} \mathrm{PI}$, and $1 \%$ (v/v) Igepal CA-630) and incubated at $37^{\circ} \mathrm{C}$ for $15 \mathrm{~min}$ in the dark. After incubation, the samples were filtered through a nylon mesh and subjected to flow cytometric analysis of PI-stained DNA fractions (Supplementary Figure $1 \mathrm{~A}$, see section on supplementary data given at the end of this article). The flow cytometry and the subsequent analyses were processed by CytoSoft Software (Millipore, Billerica, MA, USA). Fractions containing spermatocytes (tetraploid cells) and spermatids (haploid cells) with a purity above 80 and $90 \%$, respectively, were pooled together for cell slides, protein extraction, or spermatocyte culture (Supplementary Materials, Fig. 1).

\section{Immunofluorescence}

Pooled fractions were centrifuged at $500 \mathrm{~g}$ for $7 \mathrm{~min}$ and resuspended in $5 \mathrm{ml}$ of Krebs Ringer Buffer media $(120 \mathrm{mM}$ $\mathrm{NaCl}, 4.8 \mathrm{mM} \mathrm{KCl}, 25.2 \mathrm{mM} \mathrm{NaHCO}, 1.2 \mathrm{mM} \mathrm{KH_{2 }} \mathrm{PO}_{4}$, $1.2 \mathrm{mM} \mathrm{MgSO}_{4} \cdot 7 \mathrm{H}_{2} \mathrm{O}, 1.3 \mathrm{mM} \mathrm{CaCl}_{2}, 1 \times \mathrm{Pen} /$ Strep/Glu (Life Technologies), $1 \times$ essential amino acid, $1 \times$ non-essential amino acid (Lonza), and $11.1 \mathrm{mM}$ dextrose). Twenty-five microliters of the cell suspension was smeared onto a poly-Llysine microscope slide (Polysciences, Inc., 22247, Warrington, PA, USA), and the rest was subjected to protein extraction. The slides were completely air dried, and the cells were fixed with 1\% paraformaldehyde in PBS (Polysciences, Inc., 18814) for $10 \mathrm{~min}$. The cells were then washed twice with PBS before being permeabilized with $0.3 \%$ Igepal CA-630 for $10 \mathrm{~min}$. The cells were then blocked with Image-iT FX signal enhancer for $30 \mathrm{~min}$ and then rinsed with PBS. Subsequently, the cells were incubated with the appropriate antibodies in PBS containing $1 \%$ BSA for $2 \mathrm{~h}$ (Supplementary Table 1). Following a PBS wash, the cells were incubated with fluorophore-conjugated secondary antibodies diluted 1:150 in PBS containing 1\% BSA for $1 \mathrm{~h}$. The cells were then washed, and the nuclei were

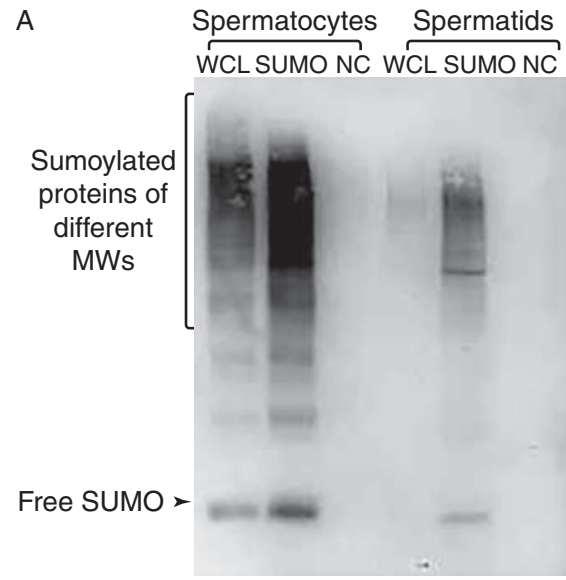

B
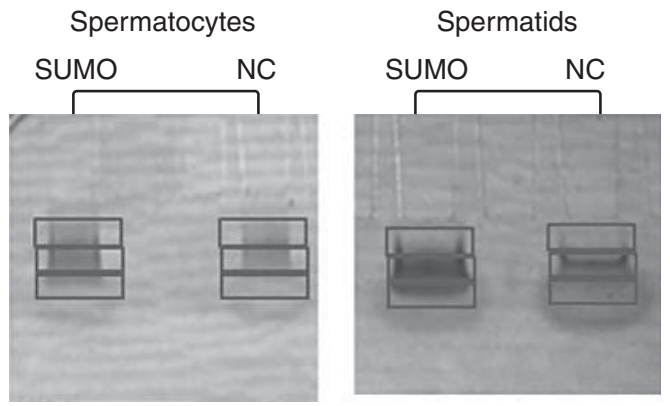

Figure 1 (A) Western blotting confirmed the successful enrichment of the IP fraction for sumoylated proteins in spermatocytes and spermatids when compared with the negative controls. Whole-cell lysate (WCL), negative control (NC), and immunoprecipitated fraction (SUMO) are shown. (B) The precipitated proteins and their corresponding negative controls were briefly run on gels, and the gels were subsequently fixed and stained. The stained regions were cut into three gel bands, digested, and analyzed by LC-MS/MS for MS.

stained with $4 \mu \mathrm{g} / \mathrm{ml}$ of 4,6-diamino-2-phenylindole (DAPI) for $5 \mathrm{~min}$. After one wash, the slides were mounted with ProLong Gold antifade reagent. The images were collected with a Nikon inverted fluorescence microscope and $60 \times$ and $100 \times$ objective lenses with DAPI, FITC, and CY-5 filter sets. At least two slides with at least 50 cells on each slide were analyzed. The images are representative of the pattern obtained for the entire slide analyzed.

\section{Protein extraction}

Both denaturing and non-denaturing lysis buffer was used for cell lysis. Many proteins are covalently and/or non-covalently modified by SUMO. It has therefore been suggested that the use of a denaturating lysis buffer containing a high percentage of SDS would have the benefits of immediately denaturing isopeptidases and eliminating non-covalent interactions with SUMO, thereby leaving only covalent SUMO binding in place (Sarge \& Park-Sarge 2009, Tatham et al. 2009, Barysch et al. 2014). The high percentage of SDS, however, would prevent sumoylated proteins from binding to anti-SUMO antibodies during the IP procedure. As a result, SDS is usually either significantly diluted $(1: 10)$ or removed from the lysis buffer by 
other means (Sarge \& Park-Sarge 2009, Tatham et al. 2009, Barysch et al. 2014). However, these manipulations can cause certain proteins to re-nature and non-covalent interactions to reform. In our studies of several SUMO targets, no significant difference was found between the results of a co-IP performed using denaturing and non-denaturing lysis buffers (Supplementary Figure 2, see section on supplementary data given at the end of this article). In both cases, the conclusions concerning possible protein sumoylation were based on the presence of high-molecular weight protein conjugate/s (one or numerous) detected with both anti-SUMO and anti-target protein antibodies above the band corresponding to the nonmodified form of the protein. The presence of a band corresponding to the molecular weight of the non-modified protein would suggest a non-covalent interaction.

To produce denatured lysates, cell pellets were re-suspended in modified $2 \times$ Laemmli buffer $(150 \mathrm{mM}$ Tris- $\mathrm{HCl} \mathrm{pH} 7.2,4 \%$ SDS, $20 \%$ glycerol, and $20 \mathrm{mM}$ N-ethylmaleimide (NEM, an isopeptidase inhibitor that blocks the activities of SENPs (Suzuki et al. 1999)) with $5 \times 10^{6}$ cells for every $300 \mu \mathrm{l}$ of buffer. The solution was sonicated until the sample become liquid and then boiled at $100{ }^{\circ} \mathrm{C}$ for $10 \mathrm{~min}$. The lysates were then collected after centrifugation at high speed at room temperature for $15 \mathrm{~min}$. The denaturing lysate was subjected to SDS removal using detergent-removal spin columns (Thermo Scientific) in accordance with the manufacturer's instructions. In brief, the SDS-containing lysate was slowly dropped onto the top of the compact resin of detergent-removal spin columns that had been washed with PBS and equilibrated and then incubated for $2 \mathrm{~min}$ at room temperature. The columns were then centrifuged at $1000 \mathrm{~g}$ for $2 \mathrm{~min}$ at room temperature, and the flow-through fractions were collected and pooled together as the SDS-removed lysate.

To produce the non-denatured lysate, $5 \times 10^{6}$ cells were re-suspended in $300 \mu \mathrm{l}$ of whole-cell extraction buffer (produced from the Millipore kit) supplemented with NEM at a final concentration of $20 \mathrm{mM}$. This cell suspension was then drawn through a 27-gauge needle five times and incubated on ice for $15 \mathrm{~min}$. The lysate was collected after a centrifugation at high speed at $4{ }^{\circ} \mathrm{C}$ for $20 \mathrm{~min}$. Lysates from five to six or two to three STA-PUT separations (for spermatocytes and spermatids respectively) were collected to produce sufficient amounts of proteins for IP (500-1300 $\mu$ g protein).

\section{Immunoprecipitation}

In all of the experiments, the lysates were pre-cleared by mixing them with control agarose resin (from Thermo Scientific, 26150) at $4{ }^{\circ} \mathrm{C}$ for $1 \mathrm{~h}$ on an orbital rotator. Each $600 \mu \mathrm{l}$ of the pre-cleared lysate $(0.5-1.3 \mathrm{mg}$ protein) was mixed with $120 \mu \mathrm{l}$ of SUMO1-agarose conjugate in a screw-cap spin column (Thermo Scientific) at $4{ }^{\circ} \mathrm{C}$. This was performed overnight on an orbital rotator and followed by centrifugation at $1400 \mathrm{~g}$ (Eppendorf, 5415C) at $4{ }^{\circ} \mathrm{C}$ for $1 \mathrm{~min}$. The amount of antibody was determined in preliminary experiments using an increasing antibody concentration and analyzing its ability to precipitate the maximal amount of sumoylated proteins from lysates containing $1 \mathrm{mg}$ protein (not shown). The retained sumoylated proteins were washed twice with whole-cell extraction buffer supplemented with $20 \mathrm{mM} \mathrm{NEM}$ and then eluted with $50 \mathrm{ml}$ acidic elution buffer (Thermo Scientific, 21004). Usage of the antibody-agarose conjugates prevented the heavy and light immunoglobulin chains from appearing in the eluted fraction, facilitating the subsequent mass spectrometry (MS) analysis.

For IP using other antibodies, the lysates were pre-cleared using protein $\mathrm{A} / \mathrm{G}$ agarose beads and incubated with the antibodies overnight (Supplementary Table 1). Mouse or rabbit IgG was used as a corresponding negative control. The lysates were washed as described above and incubated with protein A/G agarose beads overnight. This step was followed by additional washes and elution was performed as described above.

\section{Gel electrophoresis and western blotting}

Gel electrophoresis was performed using NuPAGE 4-12\% gradient bis-tris polyacrylamide gels and MOPS running buffer as previously described (Vigodner et al. 2013). The membrane (Novex nitrocellulose membrane, $0.45 \mu \mathrm{m}$ pore size, Life Technologies) was first blocked with $2 \%$ membrane blocking agent (GE Healthcare UK Limited, RPN2125V, Little Chalfont, Buckinghamshire, UK) in PBS + 0.02\% (v/v) Tween 20 (PBS-T) for $1 \mathrm{~h}$ at room temperature. The membrane was then incubated with primary antibodies in PBS containing 2\% BSA and $0.1 \%$ sodium azide for either $2 \mathrm{~h}$ at room temperature or overnight at $4{ }^{\circ} \mathrm{C}$. Following three washes with PBS-T, the membrane was further incubated with secondary antibodies that were diluted to 1:5000 in PBS-T for $1 \mathrm{~h}$ at room temperature. The secondary antibodies used in this study included the following: ECL antirabbit IgG HRP linked (GE Healthcare UK Limited, NA934V), goat anti-mouse IgG $(\mathrm{H}+\mathrm{L}) \mathrm{HRP}$ (Millipore, AP308P), and goat anti-rat IgG-HRP (Santa Cruz Biotechnology, sc-2032). The image collection and quantitative analyses were performed using the Universal Hood II and Quantity One Software (BioRad Laboratories). Depending on the quality of the images after protein separation followed by a western blot with a particular antibody, the representative images for the SUMO IP results are presented either for denaturing (cyclin-dependent kinase 1 (CDK1), serine/threonine kinase 31 (STK31), and the largest subunit of RNA polymerase II (RNAP II)) or non-denaturing (mediator of DNA damage checkpoint protein 1 (MDC1), KRAB domain-associated protein 1 (KAP1), MILI, DDX4, cell division cycle 5-like protein (CDC5), and TAR DNA-binding protein 43 (TDP-43)) protein lysates.

\section{Gel fixation and staining}

The samples to be subjected to MS analysis were run on NuPAGE 4-12\% gradient bis-tris polyacrylamide gels at $150 \mathrm{~V}$ for $5 \mathrm{~min}$. The gels were fixed using a solution containing $50 \%$ $(\mathrm{v} / \mathrm{v})$ methanol and $7 \%(\mathrm{v} / \mathrm{v})$ acetic acid at room temperature for $20 \mathrm{~min}$ and washed with distilled water three times for $5 \mathrm{~min}$ each. The fixed gels were then stained by incubation in GelCode Blue Stain Reagent (Thermo Scientific, 24590) at room temperature for $1 \mathrm{~h}$. This step was followed by washing with distilled water overnight to remove the excess staining solution. The stained regions were cut into three gel bands, digested, and analyzed by LC-MS/MS for MS. 


\section{MS analysis}

MS was performed with the assistance of the Laboratory for Macromolecular Analysis and Proteomics at the Albert Einstein College of Medicine of Yeshiva University and is described below.

In-gel trypsin digestion and nanospray LC-MS/MS was performed as described in Wang et al. (2014). Briefly, Coomassie-stained cut protein gel bands were first reduced with TCEP (tris(2-carboxyethyl)phosphine), alkylated with iodoacetamide, and digested with trypsin. Nanospray LC-MS/MS was performed using a Linear lon Trap Mass Spectrometer (Thermo Scientific) with the RSLC Chromatography System (Thermo Scientific). The ten most intense ions determined from an initial survey scan $(300-1600 \mathrm{~m} / \mathrm{z})$ were selected for fragmentation (MS/MS). The raw data files were converted to mgf text files (Mascot generic file) with Proteome Discoverer 1.2 and then merged and searched against the human or mouse NCBI database (April 2014) using the in-house Mascot Protein Search Engine (Matrix Science) with an automatic decoy database search. The following search parameters were used: trypsin, two missed cleavages; fixed modification of carbamidomethylation (Cys); variable modifications of deamidation (Asn and Gln), pyro-glu (Glu and Gln), and oxidation (Met); monoisotopic masses; peptide mass tolerance of $2 \mathrm{Da}$; and product ion mass tolerance of $0.6 \mathrm{Da}$. The Mascot-identified proteins were further validated with Scaffold (version 4, Proteome Software) using 99 and 95\% protein and peptide probability respectively, and a minimum of two peptides. The peptide and protein false discovery rates were adjusted to $1 \%$ or less.

\section{In vitro sumoylation assay}

In vitro sumoylation assays were performed with the SUMOylation Kit from Active Motif, Inc. (40120, Carlsbad, CA, USA), following the manufacturer's protocol. The mouse GST-CDK1 recombinant protein was purchased from Sino Biological, Inc. (Beijing, China).

\section{Bioinformatics analysis}

All of the putative sumoylation sites and SUMO-interaction motifs (SIMs) of the proteins were identified with SUMOsp 2.0 (The CUCKOO Workgroup, USTC). The identified proteins were divided into functional groups based on a literature search.

\section{Results}

\section{Separation of spermatocytes and spermatids}

The spermatocytes and spermatids were separated with a STA-PUT procedure that utilizes differential sedimentation velocity at the unit gravity of different cell types (Bellve et al. 1977, La Salle et al. 2009). The contents of the fractions collected from the separation were examined using microscopy and flow cytometry to identify tetraploid and haploid cells. The details of the procedure and the analysis of fraction purity are described in Supplementary Figure 1A and B.

\section{Identification of sumoylated targets}

For the identification of SUMO targets, proteins were extracted from isolated fractions using denaturing buffer followed by SDS removal and immunoprecipitated with anti-SUMO1-agarose conjugates as described in the Materials and methods section. Agarose resin without cross-linkage to antibodies was used as a negative control. Western blotting confirmed the successful enrichment of sumoylated proteins in the IP fraction compared with the negative controls (Fig. 1A). The precipitated proteins and their corresponding negative controls were then briefly run on gels, and the gels were subsequently fixed and stained (Fig. 1B). The stained regions were cut into three gel bands, digested, and analyzed by LC-MS/MS for MS. In addition to the enrichment of specific proteins, some non-specific background was observed in the negative controls. After protein digestion, MS analyses revealed $\sim 120$ proteins uniquely in the antibody fractions but none in any of the negative controls (Table 1). These identified proteins were subdivided into eight groups according to their previously published functions (Matunis et al. 1996, Moroianu 1998, Stopka et al. 2000, Goldberg et al. 2003, Myojin et al. 2004, Cramer 2006, Stark \& Taylor 2006, Bao et al. 2012, Vourekas et al. 2012, Wang et al. 2012, Lasko 2013; Fig. 2). The largest group, with $33-34 \%$ of the spermatocyte and spermatid SUMO targets, included proteins involved in transcription, RNA interaction and stability, and splicing. This group included numerous ribosomal and heterogeneous nuclear ribonucleoproteins, important splicing factors, and several novel SUMO targets with important roles in germ cells, such as the largest subunit of RNAP II, Asp-Glu-Ala-Asp (DEAD) box polypeptide DDX4 and DDX42, PIWI-like protein 2 (MILI/PIWIL2), TDP-43, and paraspeckles component 1 (Table 1 and Fig. 2).

Glycolytic and mitochondrial enzymes were found to be sumoylated in both spermatocytes (16\%) and spermatids $(10 \%)$, together with other enzymes (10-11\%). Several proteins involved in ubiquitination, including ubiquitin-activating and ubiquitin-conjugating enzymes, ubiquitin hydrolases, and proteasome subunits were identified as SUMO targets in spermatocytes $(9 \%)$ and in spermatids (3\%) (Table 1 and Fig. 2).

Stress-related and heat shock proteins represented $6-7 \%$ of sumoylated targets in spermatocytes and spermatids. Membrane-associated, vesicle trafficking, and ER proteins represented $11 \%$ of sumoylated proteins in spermatocytes and $14 \%$ in spermatids. This group included Rab7 and Rab11, calreticulin and acrosinbinding protein precursors, and the testis-specific ER protein calmegin (Table 1 and Fig. 2).

Cytoskeletal proteins represented only $2 \%$ of spermatocyte but $12 \%$ of spermatid SUMO targets. Conversely, proteins involved in DNA-break repair and chromatin remodeling were primarily sumoylated in spermatocytes 
Table 1 Identification of sumoylated proteins unique to the spermatocyte or spermatid IP samples via tandem MS. Protein ID, molecular weight, and the number of unique peptides identified for each protein in spermatocytes and/or spermatids is indicated.

\begin{tabular}{|c|c|c|c|c|}
\hline \multirow[b]{2}{*}{ Protein } & \multirow[b]{2}{*}{ Accession number ${ }^{a}$} & \multirow[b]{2}{*}{$\mathbf{M W}(\mathrm{kDa})$} & \multicolumn{2}{|c|}{ Number of unique peptides } \\
\hline & & & Spermatocytes & Spermatids \\
\hline \multicolumn{5}{|l|}{ Stress-related, heat shock proteins } \\
\hline Heat shock $70 \mathrm{kDa}$ protein 4 & gi|112293266 (+3) & 94 & 18 & 11 \\
\hline Inducible heat shock protein 70 & gi|118490060 (+4) & 70 & & 7 \\
\hline Stress-70 protein, mitochondrial & gi| $162461907(+5)$ & 73 & 5 & 4 \\
\hline Heat shock 70 kDa protein 1 -like & gi|124339838(+3) & 71 & & 2 \\
\hline Heat shock protein $105 \mathrm{kDa}$ & gi| $114145505(+5)$ & 96 & & 3 \\
\hline Glutathione $S$-transferase $\mathrm{P} 1$ & gi|10092608 (+5) & 24 & 2 & \\
\hline 1 -Cys peroxiredoxin protein 2 & gi|3789944 (+3) & 25 & 2 & \\
\hline Dnal homolog subfamily B member 1 (heat shock 40 kDa protein 1 ) & gi|9055242 (+2) & 38 & 2 & \\
\hline Protein DJ-1 & gi|55741460 (+1) & 20 & 2 & \\
\hline Ephx1 protein & gi|34784388 (+4) & 51 & 2 & \\
\hline \multicolumn{5}{|l|}{ DNA breaks, chromatin remodeling } \\
\hline Mediator of DNA damage checkpoint protein 1 (MDC1) & gil132626693 (+5) & 185 & 8 & \\
\hline SWI/SNF-related regulator of chromatin 5 (SMARCA5) & gi| $148678936(+1)$ & 116 & 5 & \\
\hline SMARCA4 & gi| $148693261(+8)$ & 185 & 2 & \\
\hline Matrin-3 & gi| $25141233(+4)$ & 95 & 3 & 3 \\
\hline ruvB-like & gi| $9790083(+1)$ & 50 & 3 & \\
\hline ruvB-like 2 & gi|6755382 (+1) & 51 & 2 & \\
\hline AT rich interactive domain 2 (ARID, RFX-like) & gi|262231796 & 196 & 3 & \\
\hline Damage specific DNA binding protein 1 & gi|148709424 (+5) & 108 & 3 & \\
\hline Poly (ADP-ribose) polymerase 1 & gi| $20806109(+4)$ & 113 & 2 & \\
\hline \multicolumn{5}{|l|}{ SUMO/ubiquitin pathway } \\
\hline Tripartite motif protein 28 (TRIM28, KAP1) SUMO ligase & gi|148706135 (+3) & 89 & 7 & 2 \\
\hline Ubgln1 protein, partial & gi| $16307349(+8)$ & 47 & 3 & \\
\hline Ubiquitin-associated protein 2-like isoform 3 & gi| $260166704(+9)$ & 117 & & \\
\hline Ubiquitin-conjugating enzyme E2 N & gi|309262615 (+3) & 23 & 2 & \\
\hline Ubiquitin-activating enzyme E1-like 2, isoform CRA_a & gi| $148706006(+4)$ & 119 & 2 & \\
\hline Ubiquitin carboxyl-terminal hydrolase isozyme L3 & gi|139948802 (+1) & 26 & 2 & \\
\hline Ubiquitin carboxy-terminal hydrolase L1 & gi|148705826 (+2) & 26 & 2 & \\
\hline Proteasome subunit alpha type 4 & gi|6755196 (+2) & 29 & 2 & \\
\hline Proteasome subunit beta type 3 & gi|6755202 & 23 & 2 & \\
\hline $26 S$ protease regulatory subunit 4 & gi| 6679501 & 49 & 2 & \\
\hline $26 \mathrm{~S}$ proteasome non-ATPase regulatory subunit 1 (Psmd1) & gi|116283726 (+5) & 93 & 2 & \\
\hline $26 \mathrm{~S}$ proteasome non-ATPase regulatory subunit 2 (Psmd2) & gi|27692965 (+8) & 67 & 2 & \\
\hline $26 \mathrm{~S}$ proteasome non-ATPase regulatory subunit 6 (Psmc6) & gi|28175479 (+3) & 44 & & 4 \\
\hline \multicolumn{5}{|l|}{ Cell-cycle regulators } \\
\hline Cyclin-dependent kinase 1 & gi|31542366 (+3) & 34 & 4 & 3 \\
\hline Serine/threonine kinase 31 & gil $13603843(+2)$ & 115 & 3 & \\
\hline Cell division cycle 5 -like protein & gi|22779899 (+3) & 92 & & 2 \\
\hline ASR2B & gi|13517493 (+3) & 100 & & 2 \\
\hline \multicolumn{5}{|l|}{ Nuclear-cytoplasmic transport } \\
\hline RAN GTPase activating protein 1 & gi|148672614 (+5) & 73 & 13 & 2 \\
\hline Ran-specific GTPase-activating protein & gi|153792001 (+2) & 24 & & 2 \\
\hline Ran binding protein 5 & gi|12057236 (+2) & 124 & 6 & 2 \\
\hline Karyopherin (importin) beta 1 & gi|30931411 (+4) & 97 & 4 & 2 \\
\hline Exportin-2 & gi|12963737 & 110 & 2 & \\
\hline \multicolumn{5}{|l|}{ Transcription, RNA-interaction/stability, splicing } \\
\hline DEAD (Asp-Glu-Ala-Asp) box polypeptide 4 & gi|148686462 (+2) & 78 & 4 & \\
\hline DEAD box polypeptide 42 & gil133777033 (+6) & 89 & 2 & \\
\hline Piwi-like protein 2 & gi|10946610 & 109 & 5 & \\
\hline Paraspeckle component 1 & gi|225543409 (+2) & 59 & 2 & \\
\hline RNA polymerase II largest subunit & gi| $2145091(+2)$ & 217 & 3 & \\
\hline RNA-binding protein EWS & gi|88853581 (+4) & 69 & 3 & 3 \\
\hline RNA-binding protein 14 & gi|86262142 (+1) & 36 & 7 & 4 \\
\hline TAR DNA-binding protein 43 isoform 1 & gi|21704096 (+4) & 45 & 2 & \\
\hline PC4 and SFRS1-interacting protein & gi|19527168 & 51 & 5 & 6 \\
\hline Elongation factor 2 & gi|33859482 (+8) & 19 & 4 & 4 \\
\hline Splicing factor, proline- and glutamine-rich & gi|23956214 (+1) & 60 & & \\
\hline Splicing factor, arginine/serine-rich 14 & gi|148696857 (+4) & 120 & 2 & \\
\hline Splicing factor $3 \mathrm{~B}$ subunit 1 & gi|15214281 (+1) & 146 & 3 & \\
\hline Splicing factor 1 protein & gi|14318588 (+10) & 67 & 6 & 2 \\
\hline Splicing factor, arginine/serine-rich 15 & gi|109150409 & & & 2 \\
\hline Far upstream element (FUSE)-binding protein 2 & gil163954948 (+2) & 75 & 7 & 9 \\
\hline Far upstream element (FUSE) binding protein 3 & gi|224922832 (+2) & 62 & & 2 \\
\hline Fusion, derived from $t(12 ; 16)$ malignant liposarcoma & gil $148685669(+6)$ & 98 & 3 & 6 \\
\hline
\end{tabular}


Table 1 Continued.

\begin{tabular}{|c|c|c|c|c|}
\hline \multirow[b]{2}{*}{ Protein } & \multirow[b]{2}{*}{ Accession number ${ }^{a}$} & \multirow[b]{2}{*}{$\mathbf{M W}(\mathrm{kDa})$} & \multicolumn{2}{|c|}{ Number of unique peptides } \\
\hline & & & Spermatocytes & Spermatids \\
\hline Interleukin enhancer-binding factor 3 isoform 1 & gil111607430 (+7) & 60 & & 3 \\
\hline T-complex protein 1 subunit epsilon & gi|6671702 (+6) & 68 & 6 & 5 \\
\hline Nonsense mRNA reducing factor 1 (NORF1) & gil12836885 (+4) & & 7 & 2 \\
\hline THO complex subunit 4 & gi|6755763(+1) & 27 & 3 & 2 \\
\hline HLA-B-associated transcript 3 & gi|148694699 (+7) & 111 & 2 & \\
\hline Polypyrimidine tract binding protein 2 & gi|148680404 (+4) & 68 & 4 & \\
\hline Prohibitin-2 & gi|126723336 (+2) & 33 & 2 & \\
\hline Abce1 protein, partial & gi|45219736 (+3) & 65 & 2 & \\
\hline Enhancer of mRNA-decapping protein 4 & gi|145566774 (+4) & 152 & 3 & \\
\hline Protein strawberry notch homolog 1 & gil $124487087(+2)$ & 154 & 3 & \\
\hline \multicolumn{5}{|l|}{ Ribonucleoproteins } \\
\hline Heterogeneous nuclear ribonucleoprotein $\mathrm{A} / \mathrm{B}$ isoform 1 & gi|146260280 (+5) & 95 & 5 & 3 \\
\hline Heterogeneous nuclear ribonucleoprotein $\mathrm{A} 1$ isoform $\mathrm{b}$ & gi|85060507 (+6) & 16 & & 6 \\
\hline Heterogeneous nuclear ribonucleoprotein $U$ & gi|148681230 (+6) & 49 & 5 & \\
\hline Heterogeneous nuclear ribonucleoprotein $\mathrm{H}$ & gi|10946928 (+2) & 123 & 2 & 5 \\
\hline Small nuclear ribonucleoprotein $\mathrm{N}$ & gi| $3142634(+2)$ & 25 & 3 & \\
\hline Heterogeneous nuclear ribonucleoprotein Q isoform 1 & gi| $114145493(+9)$ & 70 & & 4 \\
\hline Heterogeneous nuclear ribonucleoprotein $\mathrm{L}$ & gil183980004 $(+6)$ & 64 & 2 & \\
\hline Heterogeneous nuclear ribonucleoprotein $\mathrm{A} 3$ isoform a & gi|31559916 (+11) & 40 & & 2 \\
\hline Heterogeneous nuclear ribonucleoprotein r protein, & gi|13435603 (+6) & 67 & & 2 \\
\hline $116 \mathrm{kDa}$ U5 small nuclear ribonucleoprotein component isoform b & gi| $158508674(+5)$ & 109 & 2 & \\
\hline Heterogeneous nuclear ribonucleoprotein D-like & gi| $148664250(+2)$ & 46 & & 2 \\
\hline \multicolumn{5}{|l|}{ Ribosomal proteins } \\
\hline $60 S$ ribosomal protein L31-like isoform 1 & gi|82898755 (+6) & 14 & 2 & \\
\hline $60 S$ ribosomal protein L21 & gi|31560385 (+13) & 39 & 2 & \\
\hline $60 S$ ribosomal protein $\mathrm{L} 14$ & gil13385472(+1) & 24 & & 2 \\
\hline $60 S$ ribosomal protein L22 & gi| 6677775 & 15 & 2 & \\
\hline $60 S$ ribosomal protein L23-like & gi|407262287 (+2) & 14 & 2 & \\
\hline $40 S$ ribosomal protein $\mathrm{S} 13$ & gi|15029927 (+2) & 16 & 2 & \\
\hline $40 \mathrm{~S}$ ribosomal protein $\mathrm{S} 20$ isoform 2 & gi|4506697 & 13 & 2 & \\
\hline $40 S$ ribosomal protein $\mathrm{S} 19$ & gil12963511 (+3) & 16 & 2 & \\
\hline $16 S$ ribosomal protein & gi|200796 (+1) & 87 & 2 & 2 \\
\hline Ribosomal protein S26 & gil1527176 (+3) & 13 & 2 & 2 \\
\hline Ribosomal protein S9 & gi| $21594169(+1)$ & 23 & 3 & 2 \\
\hline Ribosomal protein S23 & gi|72679974 (+5) & 16 & 2 & \\
\hline La ribonucleoprotein domain family member 1 & gi| $147744571(+3)$ & 121 & & 2 \\
\hline \multicolumn{5}{|l|}{ Cytoskeleton } \\
\hline Lamin-B1 & gi|188219589 (+2) & 67 & 3 & 6 \\
\hline Alpha-actinin-4 & gi|11230802 & 105 & 2 & \\
\hline Filamin-C & gi|124487139 (+2) & 291 & & 10 \\
\hline Filamin-B & gi|145966915 (+2) & 277 & & 8 \\
\hline Talin 1 & gi| $148670519(+4)$ & 270 & & 3 \\
\hline Vinculin & gil148669535 (+3) & 124 & & 3 \\
\hline Coiled-coil domain containing 39 & gi| $148703084(+2)$ & 107 & 2 & \\
\hline Kinesin family member $5 \mathrm{~B}$ & gil148691088 $(+3)$ & 110 & & 4 \\
\hline Protein syndesmos & gil13385314 (+1) & 23 & & 3 \\
\hline Plectin & gi|122065897 (+24) & 534 & & 2 \\
\hline Myosin light chain, regulatory B-like & gi|71037403 (+4) & 20 & & 3 \\
\hline \multicolumn{5}{|l|}{ Membrane-associated, vesicle trafficking, ER proteins } \\
\hline Acrosin-binding protein isoform 1 precursor & gi|188035922(+1) & 61 & 2 & \\
\hline GPI-anchored membrane protein 1 & gil148695758 (+7) & 84 & 4 & 4 \\
\hline Ras-related protein Rab-11B & gi|6679583 (+3) & 24 & 5 & 2 \\
\hline Ribophorin I & gi|148666824 (+4) & 68 & & 2 \\
\hline Ribophorin & gi|1468961 (+4) & 66 & 7 & 2 \\
\hline Calreticulin precursor & gi|6680836 (+5) & 48 & & 2 \\
\hline Rab7 & gi|1050551 (+1) & 24 & 3 & 2 \\
\hline Programmed cell death 6 interacting protein & gi|20071292(+8) & 96 & 4 & 2 \\
\hline Calmegin, isoform CRA_b & gil148678956 (+3) & 71 & 3 & 2 \\
\hline Calnexin precursor & gi|6671664 (+1) & 67 & 2 & \\
\hline Solute carrier family 2, facilitated glucose transporter member 3 & gi|261862282(+2) & 53 & 3 & 3 \\
\hline Transmembrane emp24-like trafficking protein 10 & gil148670919 $(+2)$ & 26 & & 2 \\
\hline Ras-related protein Rab-14 & gi|18390323 (+3) & 24 & 3 & \\
\hline SEC22 vesicle trafficking protein homolog B & gil14290512(+1) & 25 & 2 & \\
\hline ERO1-like beta & gil109730421 (+3) & 54 & 2 & \\
\hline p162 protein & gi|1205976 (+7) & 162 & 2 & \\
\hline Zinc finger protein 289 & gi|148695611 (+4) & 58 & 2 & \\
\hline
\end{tabular}


Table 1 Continued.

\begin{tabular}{|c|c|c|c|c|}
\hline \multirow[b]{2}{*}{ Protein } & \multirow[b]{2}{*}{ Accession number $^{\mathrm{a}}$} & \multirow[b]{2}{*}{$\mathbf{M W}(\mathrm{kDa})$} & \multicolumn{2}{|c|}{ Number of unique peptides } \\
\hline & & & Spermatocytes & Spermatids \\
\hline \multicolumn{5}{|l|}{ Glycolitic and mitochondria enzymes } \\
\hline Cytochrome $c$ oxidase subunit $6 \mathrm{C}$ & gi| 16716343 & 8 & 2 & \\
\hline Cytochrome b-c1 complex subunit 2 , mitochondrial precursor & gi|22267442 (+2) & 48 & 2 & \\
\hline Cytochrome b-C1 complex subunit 1 , mitochondrial precursor & gi|46593021 (+3) & 53 & 3 & \\
\hline Cytochrome $c$ oxidase subunit IV & gi|1372988 (+2) & 20 & 3 & \\
\hline Dihydrolipoamide $S$-acetyltransferase precursor & gi|16580128 (+2) & 59 & 3 & \\
\hline Trifunctional enzyme subunit alpha, mitochondrial precursor & gi|33859811 (+1) & 83 & 6 & \\
\hline Trifunctional enzyme subunit beta, mitochondrial precursor & gi|21704100 (+4) & 51 & 2 & \\
\hline Phosphoglycerate mutase 1 & gi|114326546 (+1) & 29 & & 2 \\
\hline L-lactate dehydrogenase A chain isoform 2 & gi|257743039 (+5) & 40 & 3 & 2 \\
\hline L-lactate dehydrogenase B chain & gi|6678674 & 37 & 2 & 2 \\
\hline Phosphate carrier protein, mitochondrial precursor & gi|19526818 (+6) & 40 & 2 & \\
\hline Fructose-bisphosphate aldolase $\mathrm{A}$ isoform 1 precursor & gi|293597567 (+2) & 45 & & 5 \\
\hline Aldehyde dehydrogenase 2, mitochondria & gi|148687772 (+5) & 55 & & 4 \\
\hline Me1 protein & gi|13096987 (+6) & 64 & 2 & 2 \\
\hline ATP synthase subunit $O$, mitochondrial precursor & gi|20070412 (+2) & 23 & 3 & \\
\hline Inositol-3-phosphate synthase 1 & gi|12963757 & 61 & 2 & \\
\hline Inner membrane protein, mitochondrial & gi|148666538 (+10) & 81 & 2 & \\
\hline Creatine kinase B-type & gi|10946574 (+3) & 43 & & 3 \\
\hline Acyl-CoA hydrolase & gi|14587839 (+6) & 38 & 2 & \\
\hline$N(4)$-(beta- $N$-acetylglucosaminyl)-L-asparaginase isoform 1 precursor & gil54292135 & 37 & 3 & \\
\hline Carnitine $O$-palmitoyltransferase 2 , mitochondrial precursor & gi|162138915 (+3) & 74 & 2 & \\
\hline Isocitrate dehydrogenase $3(\mathrm{NAD}+)$ alpha & gi|148693872(+5) & 40 & 2 & \\
\hline Enoyl-CoA delta isomerase 1, mitochondrial precursor & gi|31981810 (+1) & 32 & 2 & \\
\hline Coiled-coil-helix-coiled-coil-helix domain containing 3 & gi|148681756 (+2) & 23 & 2 & \\
\hline \multicolumn{5}{|l|}{ Other enzymes } \\
\hline GMP synthase (glutamine-hydrolyzing) & gi|85861218 (+4) & 77 & 2 & \\
\hline Tripeptidyl peptidase II & gi|148664483 (+4) & 139 & 2 & \\
\hline Aspartyl-tRNA synthetase & gi|14250408 (+6) & 57 & 2 & \\
\hline Triosephosphate isomerase, partial & gi|1864018 (+5) & 23 & & 4 \\
\hline Retinol dehydrogenase 11 precursor & gi|19482172(+3) & 35 & & 2 \\
\hline Hydroxysteroid $(17-\beta)$ dehydrogenase 4 & gi|148677986 (+3) & 33 & 3 & \\
\hline \multicolumn{5}{|l|}{ Immunoglobulins } \\
\hline Ig heavy chain $\mathrm{V}$ region TE32 & gil110285 (+14) & 13 & 2 & 2 \\
\hline Kappa-Ig light chain (111 AA) & gi|930228 & 12 & 9 & \\
\hline \multicolumn{5}{|l|}{ Unknown functions in testis } \\
\hline Ataxin 2-like & gi|148685438 & 113 & 10 & 4 \\
\hline Testis specific 10 & gi|148682582 (+2) & 80 & 4 & \\
\hline Neuroleukin & gi|200065 (+8) & 63 & 2 & 2 \\
\hline Myelin expression factor 2 isoform 1 & gi|244790087 (+4) & 63 & 2 & \\
\hline Platelet-activating factor acetylhydrolase IB subunit gamma & gi|6679201 & 26 & 2 & \\
\hline Interleukin enhancer-binding factor 2 & gi|13385872 (+2) & 43 & 2 & \\
\hline Tetratricopeptide repeat protein 21B & gi|114158711 (+1) & 151 & 2 & \\
\hline Annexin $\mathrm{A} 3$ & gi|148688409 (+6) & 36 & & 2 \\
\hline HDL-binding protein & gi|148708002 (+5) & 144 & & 2 \\
\hline
\end{tabular}

'The number showing in the bracket following ' + ' means how many more accession number also refer to this target.

(7\%) but not in spermatids (1\%). Importantly, this group included several proteins implicated in the regulation of chromatin remodeling during meiosis, such as MDC1, SWI/SNF-related regulator of chromatin 4 and 5 (SMARCA4 and SMARCA5), and poly (ADP-ribose) polymerase 1 (Table 1 and Fig. 2).

Proteins that mediate nuclear-cytoplasmic transport comprised $3 \%$ of the spermatocyte and $5 \%$ of the spermatid sumoylome. Importantly, the major known sumoylated target in cells, Ran GTPase activating protein 1 (RanGAP1), was identified in both spermatocyte and spermatid fractions. This finding serves as a positive control for the specificity of SUMO target identification. Other proteins in this group included Ran binding protein 5, importin beta, and exportin 2, which are known to interact with RanGAP1 (Roscioli et al. 2012).

Cell-cycle regulators represented 2 and $4 \%$ of sumoylated proteins in spermatocytes and spermatids respectively. These important regulators of meiosis and mitosis include CDK1 (CDC2), testis-specific STK31, and CDC5 (Table 1 and Fig. 2).

\section{Confirmation of possible sumoylated targets}

GPS-SUMO, a tool for the prediction of sumoylation sites and SIMs, was used to identify consensus and non-consensus sequences for possible sumoylation within the amino acid sequences of the proteins 
A
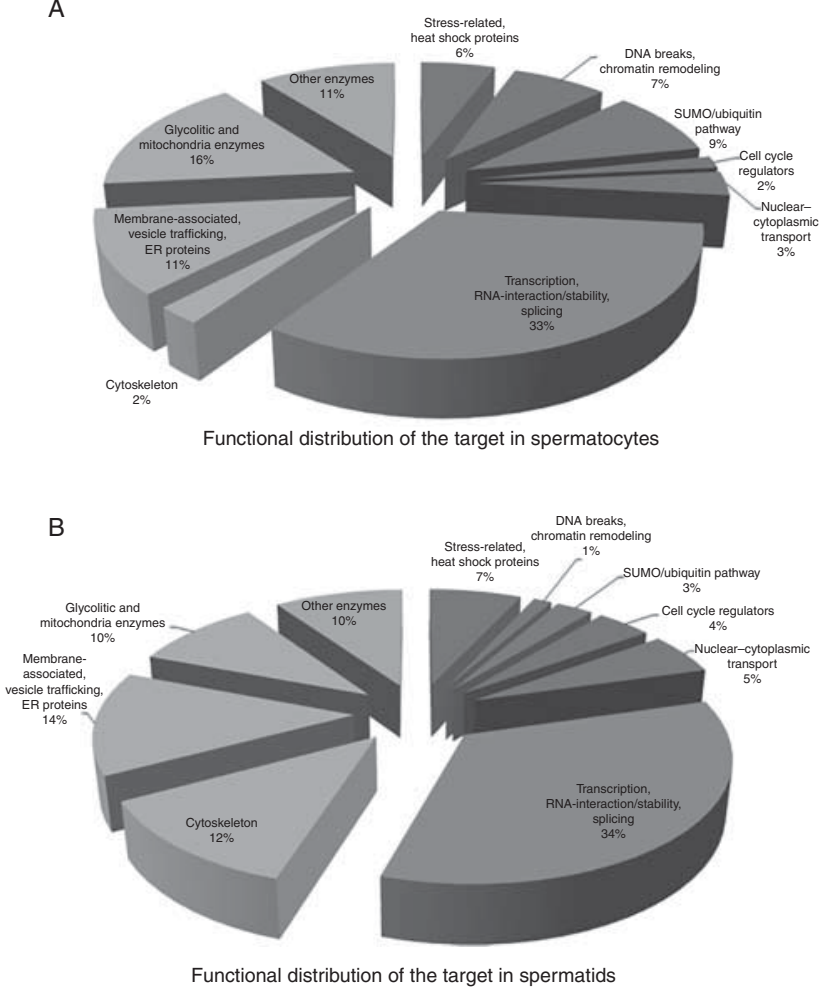

Figure 2 Functional distribution of SUMO targets in mouse spermatocytes (A) and spermatids (B). The percentage of sumoylated proteins from a certain functional group out of $100 \%$ of the identified sumoylated proteins is shown.

(Zhao et al. 2014). Because $\sim 40 \%$ of proteins that are sumoylated are not sumoylated within the consensus sequence, the prediction algorithm of GPS-SUMO is based on the analysis of 983 manually collected consensus and non-consensus sumoylation sites in 545 proteins and 137 known SIMs in 80 proteins (Zhao et al. 2014). Several proteins with important roles during spermatogenesis (displaying an infertility phenotype upon inactivation or/and germ cell-specific proteins) were analyzed for the presence of sumoylation sites, and several of those with multiple and/or conserved sites were chosen for further characterization. These proteins included MDC1, KAP1, MILI, DDX4, CDK1, CDC5, STK31, TDP-43, and the largest subunit of RNAP II. Co-IP assays performed with anti-SUMO1 and anti-target protein antibodies alongside their negative controls were followed by western blot analyses with antibodies against the target proteins or SUMO.

\section{RNA polymerase II}

RNAP II is a master regulator of transcription in both germ and somatic cells. RNAP II was identified as SUMO-interacting protein specifically in the spermatocyte fraction MS screen (Table 1). In lysates from whole testis lysate, purified spermatocytes, spermatids, and
HEK cells, an isoform of RNAP II was specifically identified with an anti-SUMO antibody followed by western blotting with an antibody against the largest subunit of RNAP II, suggesting that this is a sumoylated isoform (Fig. 3A). IP with an anti-RNAP II antibody did not successfully enrich for sumoylated isoforms of the proteins (not shown), which was likely due to the less efficient recognition by the antibody of the sumoylated isoform of the protein compared with the non-sumoylated form. Bioinformatics analysis revealed the presence of only non-consensus sumoylation sites in the amino acid sequence of RNAP II. However, the two sequences were evolutionary conserved between mouse and human (Supplementary Table 2, see section on supplementary data given at the end of this article).

\section{Mediator of DNA damage checkpoint protein 1}

MDC1 is an important regulator of the DNA damage response. Male-specific infertility in $M d c 1^{-1-}$ mice is due to meiotic arrest (Lou et al. 2006). MDC1 is an essential factor for establishing sex chromosome-wide silencing in the pachytene stage (Kunin et al. 2010, Ichijima et al. 2011). MDC1 was specifically identified in the spermatocyte fraction in our MS screen (Table 1). Several MDC1 isoforms in the range of $160-260 \mathrm{kDa}$ that may correspond to splice isoforms of the protein were pulled down using co-IP with anti-SUMO antibody followed by MDC1 western blot from the whole testis, purified spermatocyte but not spermatid fraction lysate (Fig. 3B). Sumoylation of MDC1 in somatic cells was previously reported (Yin et al. 2012). Bioinformatics analysis revealed the presence of several consensus sumoylation sites in both the mouse and human amino acid sequences of MDC1 (Supplementary Table 2).

\section{MILI}

MILI, a mammalian member of the Piwi gene family, binds to piRNAs. Spermatogenesis in mili-null mice is blocked at early prophase of the first meiosis, and the mice are sterile (Kuramochi-Miyagawa et al. 2004). MILI was specifically identified in the spermatocyte fraction in the MS screen (Table 1). IP with an anti-SUMO antibody followed by western blotting with an anti-MILI antibody identified an apparent non-covalent interaction of the protein with SUMO or sumoylated proteins in spermatocytes and whole testis lysate (a band of $\sim 110 \mathrm{kDa}$, Fig. 3C). The signal in spermatid fraction was very weak suggesting either an absence or a low level of SUMO and MILI interaction in these cells. Higher molecular weight (and presumably sumoylated) isoforms of MILI precipitated better with an anti-MILI antibody than with the anti-SUMO antibody followed by western blotting with either the anti-SUMO or anti-MILI antibody. Overall, these results suggest that there is both covalent and noncovalent modification of MILI by SUMO. MILI is a germ cell-specific protein, and therefore somatic data are not 
A RNAP II

IP:SUMO: WB:RNAP ||

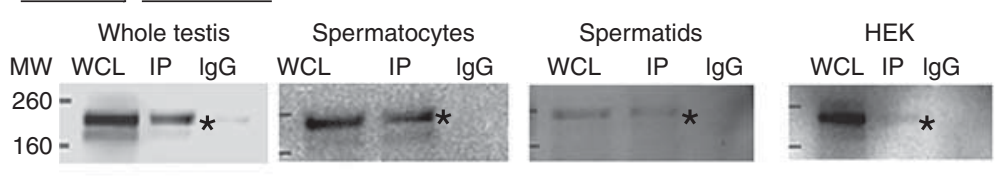

B $\quad$ MDC1

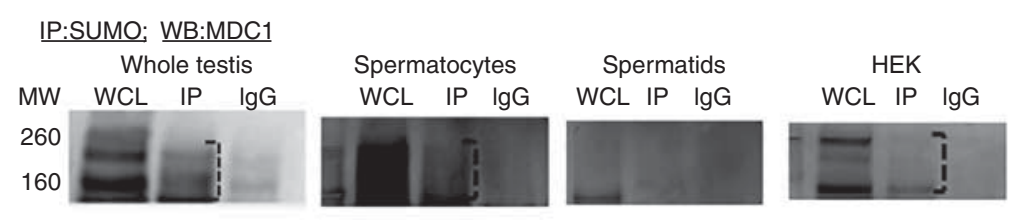

C MILI

IP:SUMO: WB:MILI

Whole testis

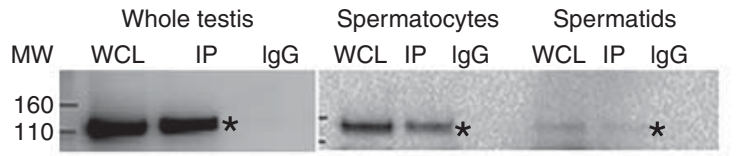

D $\underline{\text { DDX4 }}$

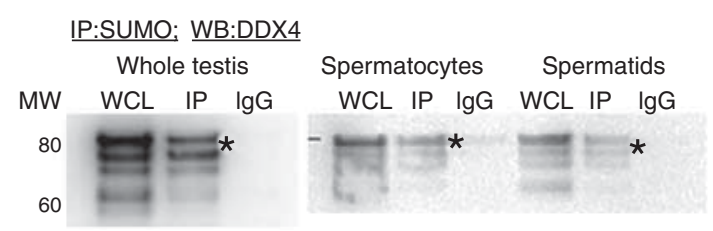

HEK

Germ cellspecific
HEK

Germ cellspecific

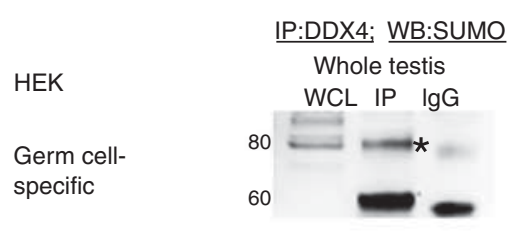

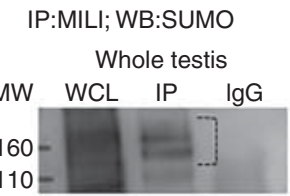
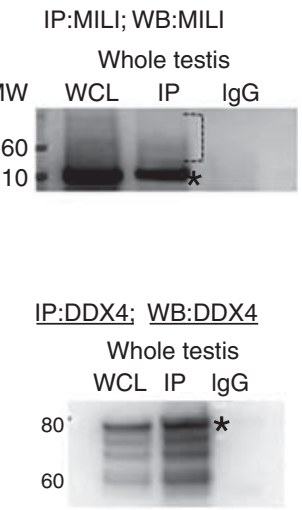

Figure 3 Co-IP analysis of SUMO and RNAP II (A), MDC1 (B), MILI (C), and DDX4 (D). Whole-cell lysate (WCL), negative control (IgG), and IP fractions are shown. The migrating positions of the molecular weight (MW) markers are indicated. (A) Co-IP analysis of SUMO and RNAP II. In lysates prepared using denaturing buffer from whole testis, purified spermatocytes, spermatids, and human embryonic kidney 293 (HEK-293) cells, two isoforms of RNAP II were identified. The isoform with a higher molecular weight (asterisk) was identified by IP with an anti-SUMO antibody followed by western blotting with an anti-RNAP II antibody. (B) Using whole testis, purified spermatocytes, and HEK-293 cells lysate prepared using non-denaturing buffer, IP with an anti-SUMO antibody followed by western blotting with an MDC1 antibody detected multiple isoforms of MDC1 (bracket). There was no specific signal identified in the spermatid fraction. (C) IP using anti-SUMO or anti-MILI antibodies followed by western blotting with either anti-MILI or anti-SUMO antibodies. Using spermatocytes, spermatids, and whole testis lysate prepared using non-denaturing buffer, IP with an anti-SUMO antibody followed by western blotting with an anti-MILI antibody identified a non-covalent interaction of the protein with SUMO or sumoylated proteins (a band $\sim 110 \mathrm{kDa}$; asterisk). The signal in spermatids was at a low level. Sumoylated conjugates of higher molecular weight were precipitated with an anti-MILI antibody followed by western blotting with either the anti-SUMO or antiMILI antibody. (D) IP using SUMO or DDX4 antibodies followed by western blotting with either DDX4 or SUMO antibodies. Several isoforms of the protein could be detected on the western blot using an anti-DDX4 antibody and non-denaturing lysis buffer. The largest isoform ( $\sim 80 \mathrm{kDa}$, asterisk) could be a sumoylated form of the protein, as it was specifically identified by reciprocal co-IP using both SUMO and DDX4 antibodies.

shown. Bioinformatics analysis revealed the presence of several consensus sumoylation sites in both the mouse and human amino acid sequences of the protein (Supplementary Table 2).

\section{DDX4}

DDX4 is the mouse VASA homologue $(\mathrm{MVH})$ that is expressed exclusively in germ cells. It interacts with MILI to regulate microRNA-mediated RNA silencing (Kuramochi-Miyagawa et al. 2004). DDX4 is required for the development of male germ cells. Male mice that are homozygous for a targeted mutation of $M v h$ produce no sperm in the testes, with spermatogenic arrest at early meiosis in a manner similar to MILI-deficient mice (Tanaka et al. 2000). Several isoforms of the protein ranging from 60 to $80 \mathrm{kDa}$ were detected by western blotting using an anti-DDX4 antibody in spermatocytes, spermatids, and whole testis lysate. These proteins may be the result of alternative splicing (Luo et al. 2013; Fig. 3D). The largest isoform $(\sim 80 \mathrm{kDa})$ may be a sumoylated form of the protein, as this isoform was specifically identified by reciprocal co-IP using both SUMO and DDX4 antibodies. Although DDX4 was identified in spermatocyte fraction in the MS screen (Table 1), a more sensitive approach of western blotting 
supported sumoylation of DDX4 in both spermatocytes and spermatids. DDX4 is a germ cell-specific protein, and therefore somatic data are not shown. Bioinformatics analyses revealed the presence of several consensus sumoylation sites in both the mouse and human amino acid sequences of DDX4, supporting possible sumoylation (Supplementary Table 2).

\section{KRAB domain-associated protein 1}

KAP1 (TIF1 $\beta$, TRIM28) is a transcriptional repressor known to play essential roles in chromatin remodeling in early embryonic development and spermatogenesis. During spermatogenesis, KAP1 is preferentially associated with the heterochromatin structures of spermatocytes, spermatids, and Sertoli cells. KAPI was identified in both the spermatocyte and spermatid fractions in the MS screen (Table 1). IP with an anti-SUMO antibody followed by KAP1 western blot analysis identified a possible non-covalent interaction of the protein with SUMO (a band at $\sim 100 \mathrm{kDa}$, Fig. 4A) in the whole testis, spermatocyte, spermatid, and HEK cell lysate. Higher molecular weight SUMO-conjugates were clearly detected by IP with an anti-KAP1 antibody followed by western blotting with either an anti-SUMO or anti-KAP1 antibody (Fig. 4A). KAP1 is an important protein in Sertoli cells. Therefore, to further evaluate the possible sumoylation of KAP1 in those cells, we also employed primary human and transformed mouse Sertoli cell lines. In all Sertoli cells, co-IP analyses revealed possible covalent and non-covalent interaction of KAP1 with SUMO (Fig. 4B, human and mouse Sertoli cell lines are shown). These results suggest that Sertoli cell lines can be used to a certain degree to study the regulation of sumoylation in Sertoli cells. Sumoylation of
KAP1 in somatic cells was previously reported (Li et al. 2007, Campbell \& Izumiya 2012). Bioinformatics analysis revealed the presence of several consensus sumoylation sites in both the mouse and human amino acid sequences of the protein (Supplementary Table 2).

\section{Cyclin-dependent kinase 1}

CDK1 (CDC2) is a crucial and indispensable regulator of both mitosis and meiosis (Diril et al. 2012). CDK1 was identified in both the spermatocyte and spermatid fractions in the MS screen (Table 1). Lysates from the whole testis and purified spermatocytes and spermatids were obtained, and two isoforms of CDK1 were identified in the SUMO pull-down from the whole testis and spermatocytes, suggesting both the covalent and non-covalent interaction of CDK1 with SUMO (Fig. 5A). Only one isoform was observed at the detectable level in the spermatid and HEK cell lysate. The higher-molecular weight isoform was also highly enriched after IP with an anti-CDK1 antibody followed by western blotting with an anti-SUMO antibody (Fig. 5A). Some background signal was detected in the negative control when highly concentrated lysates were used for the CDK1 IP analysis (whole testis). However, the background was significantly lower than the specific signal and was not detected upon IP with anti-SUMO antibodies. To confirm the possible sumoylation of CDK1 and given the importance of the protein in both germ and somatic cells, an in vitro sumoylation reaction was performed with a commercially available recombinant GST-CDK1 protein, sumoylation enzymes (E1 and E2), and either normal (N) SUMO or a mutant (M) SUMO incapable of forming an isopeptide bond (Fig. 5B). Western blot analysis with an anti-CDK1 antibody revealed the

\section{A KAP1, Testis}
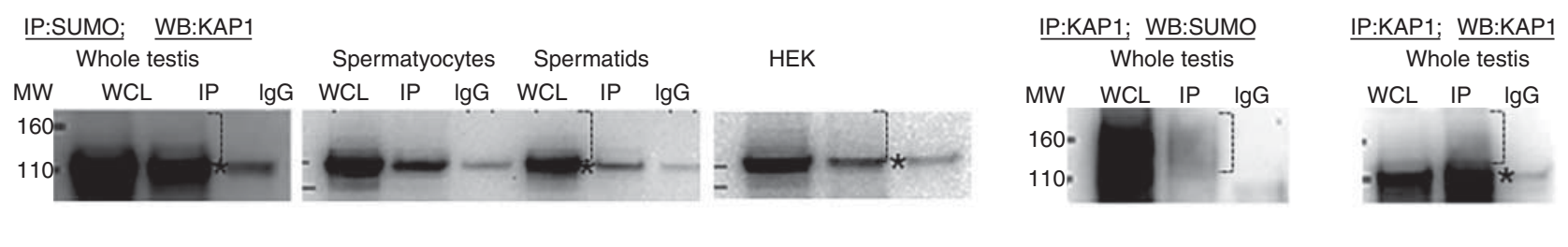

B

$\underline{\text { KAP1, Sertoli }}$

Sertoli cell line

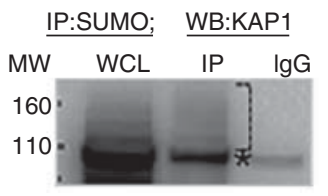

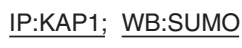

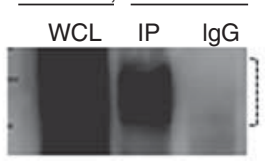

Human sertoli

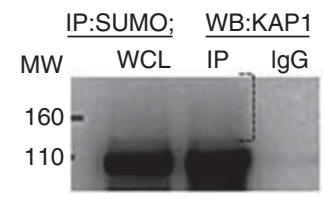

IP:KAP1; WB:SUMO

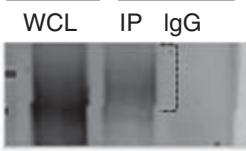

Figure 4 Co-IP analysis of SUMO and KAP1. IP using non-denaturing lysis buffer with anti-SUMO antibody followed by KAP1 western blot analysis identified a possible non-covalent interaction of the protein with SUMO (a band $\sim 100 \mathrm{kDa}$, asterisk, A). Higher molecular weight SUMO-conjugates were clearly detected by IP with an anti-KAP1 antibody followed by western blotting with either an anti-SUMO or anti-KAP1 antibody (bracket). In a similar manner, co-IPanalyses of KAP1 and SUMO in Sertoli cells and cell lines (B) revealed possible covalent and non-covalent interactions of KAP1 with SUMO. 
A $\quad \underline{C D K 1}$
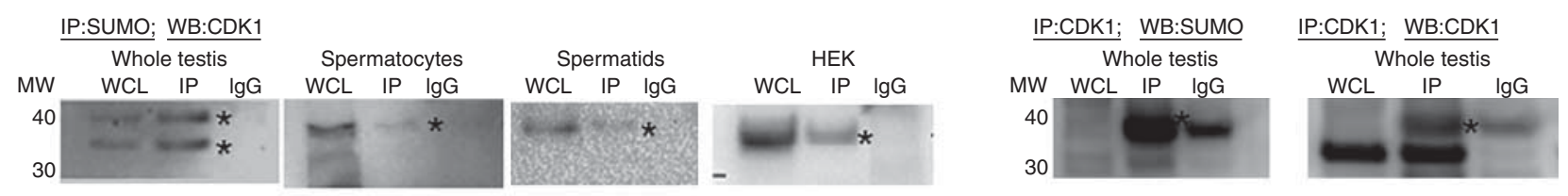

B CDK1, In vitro sumoylation

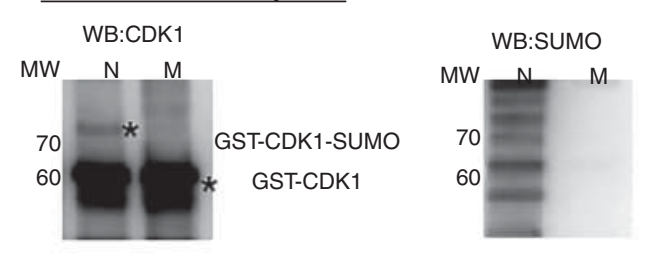

C $\quad$ CDC5

IP:SUMO; WB:CDC5
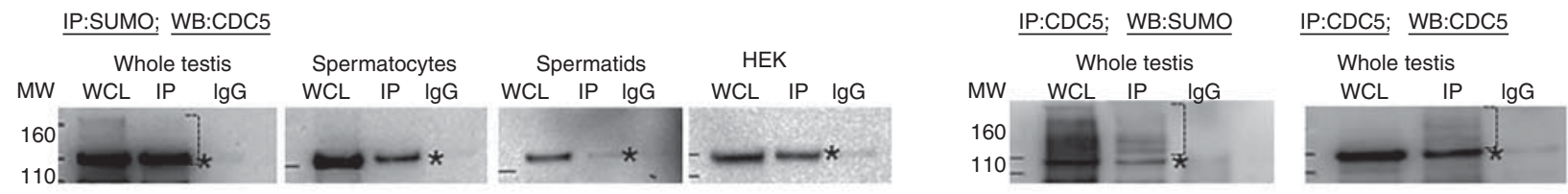

\section{D $\quad$ STK31}

\section{IP:SUMO; WB:STK31}
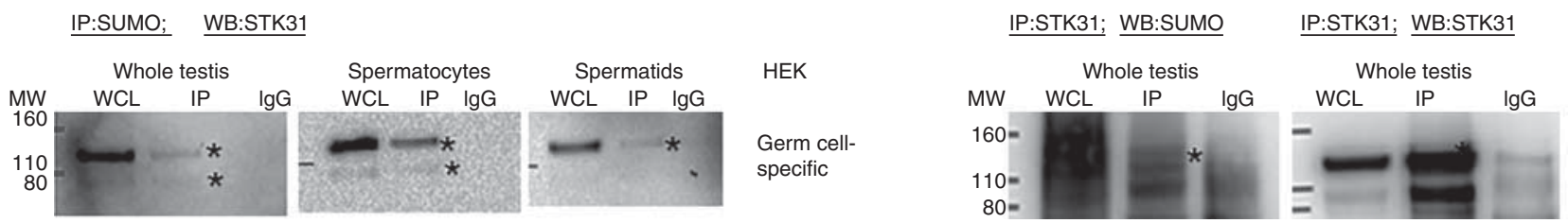

Figure 5 Co-IP analysis of SUMO and CDK1 (A), CDC5 (C), and STK31 (D) and the in vitro sumoylation analysis of CDK1 (B). (A) Co-IP analysis of SUMO and CDK1. Lysates prepared using denaturing lysis buffer from whole testis and purified spermatocytes were obtained, and two isoforms of CDK1 were identified in the SUMO pulldown, suggesting both the covalent and non-covalent interaction of CDK1 with SUMO. Only one isoform was detectable in spermatids and HEK cells. The higher-molecular weight isoform (asterisk) was also highly enriched after IP with an anti-CDK1 antibody followed by western blotting with an anti-SUMO antibody. (B) To confirm the possible sumoylation of CDK1, an in vitro sumoylation reaction was performed with a recombinant GST-CDK1 protein, sumoylation enzymes (E1 and E2), and either normal (N) or a mutant (M) SUMO incapable of forming an isopeptide bond. Western blot analysis with an anti-CDK1 antibody revealed the presence of a sumoylated CDK1 band above the non-modified GST-CDK1 (an asterisk in N and $\mathrm{M}$ lines, respectively) when using the normal but not mutant SUMO isoform. When detecting with an anti-SUMO antibody, multiple bands were observed only in the sample with normal SUMO, most likely corresponding to sumoylated E1 and E2 in addition to sumoylated CDK1. (C) Reciprocal co-IP using non-denaturing lysis buffer, anti-SUMO and anti-CDC5 antibodies support both covalent (bracket) and non-covalent interactions of CDC5 with SUMO (asterisk). (D) Co-IP analysis using non-denaturing lysis buffer, anti-SUMO, and anti-STK31 antibodies supported possible non-covalent and covalent interactions of StkTK31 with SUMO. The asterisk indicates a possible sumoylated isoform found above the non-sumoylated isoform of STK31.

presence of a sumoylated CDK1 band above the nonmodified GST-CDK1 when using the normal but not the mutant SUMO isoform (Fig. 5B). When detected with an anti-SUMO antibody, multiple bands were observed in the sample with normal SUMO, most likely corresponding to sumoylated E1 and E2 in addition to sumoylated CDK1. However, these bands were not observed in the sample with the mutant SUMO isoform. Bioinformatics analysis revealed the presence of the consensus sumoylation site in the amino acid sequence of the mouse but not the human CDK1 (Supplementary Table 2). However, the alignment of the two sequences revealed a difference in only one amino acid, with a possible target lysine still present at the same position. We examined whether another important cell-cycle regulator, CDK2 (not identified by our screen), contains a consensus sequence for sumoylation and detected no such sequence in CDK2 (not shown).

\section{Cell division cycle 5-like protein}

CDC5 is a DNA-binding protein involved in cell-cycle control. Using lysates from the whole testis, spermatocytes, spermatids, and HEK cells, reciprocal co-IP using anti-SUMO and anti-CDC5 antibodies supported the mostly non-covalent interactions of CDC5 with SUMO 
(Fig. 5C, a band $\sim 110 \mathrm{kDa}$ ) but also some weak bands of higher molecular weight, which can correspond to sumoylated isoforms of the protein (brackets). Although CDC5 was identified in the spermatide fraction in the MS screen, western blotting supported interaction between CDC5 and SUMO in both spermatocytes and spermatids. Bioinformatics analysis revealed the presence of two consensus sumoylation sites conserved between mouse and human in the amino acid sequences of CDC5 (Supplementary Table 2).

\section{Serine/threonine kinase 31}

StkTK31 is a germ cell-specific protein kinase. StkTK31 was identified in both the spermatocyte and spermatid fractions in the MS screen (Table 1). Co-IP analysis with anti-SUMO and anti-STK31 antibodies using the whole testis, spermatocyte, and spermatid lysates supported mostly covalent (Fig. 5D, a band at $\sim 110 \mathrm{kDa}$ ) and some non-covalent (a band just below $80 \mathrm{kDa}$ in some fractions) interactions of STK31 with SUMO (Fig. 5D). Although STK31 was only identified in the spermatocyte fraction in the MS screen, western blotting supported possible interaction between CDC5 and SUMO in both spermatocytes and spermatids. STK31 is a germ cell-specific protein, and somatic data are not shown. Bioinformatics analysis revealed the presence of multiple conserved consensus sumoylation sites in the amino acid sequences of STK31 in mouse and human (Supplementary Table 2).

\section{TAR DNA-binding protein 43}

TDP-43 is an evolutionarily conserved, ubiquitously expressed DNA/RNA-binding protein. In testis, it binds to the promoter of the testis-specific mouse Acrv1 gene in spermatocytes and spermatids, but ACRV1 is expressed exclusively in spermatids. Mutations in the TDP-43 promoter-binding motifs lead to the premature transcription of Acrv1 in spermatocytes. TDP-43 may be involved in pausing RNAPII at the Acrv1 promoter in spermatocytes (Lalmansingh et al. 2011). One mechanism regulating the different activity of TDP-43 in spermatocytes and spermatids could be a PTM. Our MS screen identified TDP-43 as SUMO target in spermatocytes but not in the spermatid fraction (Table 1). Notably, IP with an anti-SUMO antibody followed by TDP-43 western blot analysis of the whole testis and purified germ cell fractions (Fig. 6A) confirmed that SUMO and TDP-43 interact specifically in spermatocytes but not in spermatids (Fig. 6A and B; two membranes with increasing amounts of protein are shown). The purity of the fractions was confirmed using an anti-SYCP3 antibody showing a prominent band specifically in the spermatocyte fraction (Fig. 6B, SYCP3). Interestingly, two bands were identified using IP in HEK cells with an anti-SUMO antibody followed by TDP-43 western blot analysis, suggesting both covalent and non-covalent interactions between TDP-43 and SUMO. Bioinformatics analysis revealed the presence of a conserved non-consensus sequence at the same residues in the mouse and human proteins (Supplementary Table 2).

\section{Co-localization studies}

Partial co-localization of SUMO with its putative targets (orange signal) supported their possible sumoylation. Several targets showed a certain degree of co-localization between SUMO in the large heterochromatic regions of spermatocytes (MDC1 in the XY body

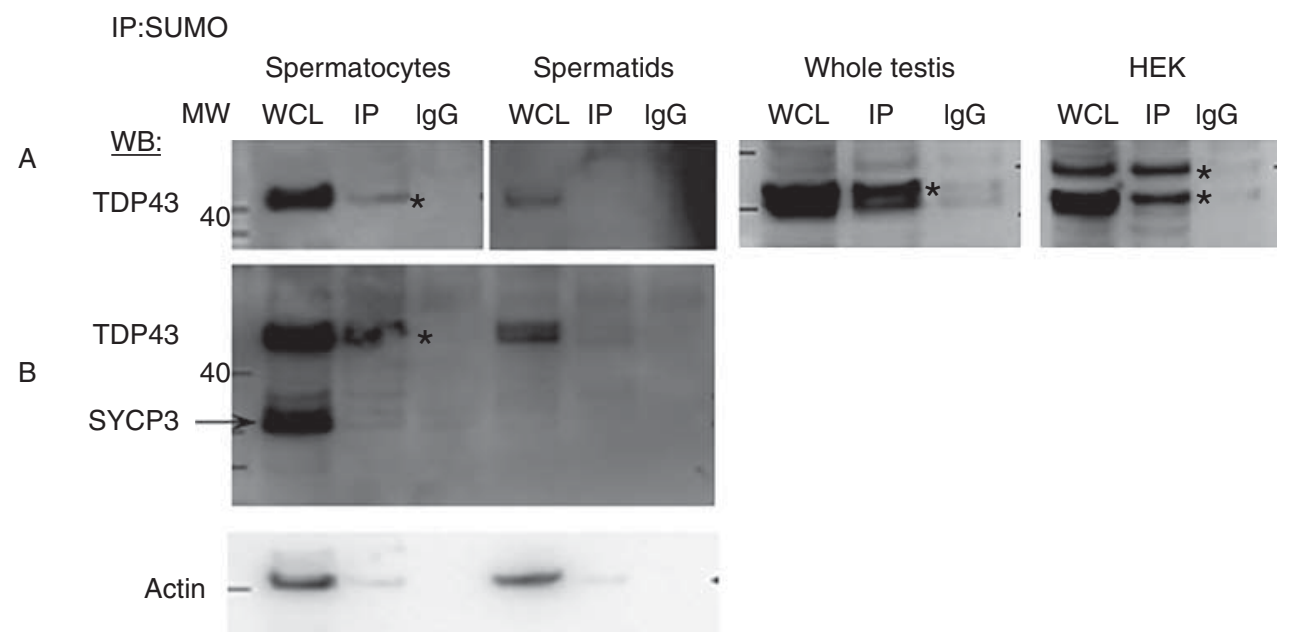

Figure 6 Co-IP analysis of SUMO and TDP-43. IP using non-denaturing lysis buffer with anti-SUMO antibody followed by TDP-43 western blot analysis identified a prominent specific signal (asterisk) in the whole testis and spermatocyte but not in the spermatid fractions (A and B show two different membranes with increasing amount of proteins). The same membrane was re-probed with anti-SYCP3 antibody (SYCP3) to confirm the purity of the spermatocyte and spermatid fractions and with an actin antibody to show a comparable level of the whole-cell lysates used for the IP. Two bands were identified in HEK cells (asterisks), suggesting both covalent and non-covalent interactions between TDP-43 and SUMO. 
(Fig. $7 \mathrm{~A}_{1}$ and $\mathrm{A}_{2}$, arrowheads) and KAP1 in the centromeric heterochromatin and partially in the $X Y$ body of spermatocytes (arrow and arrowheads respectively, Fig. $7 \mathrm{~B}_{1}$ and $\mathrm{B}_{2}$ ) and chromocenters of round spermatids (Fig. $7 \mathrm{C}_{1}$ and $\mathrm{C}_{2}$, arrowheads). KAP1 also co-localized with SUMO in certain areas of human and mouse Sertoli cells as well as in mouse Sertoli cell lines (Fig. 7D $, D_{2}, E_{1}$ and $E_{2}$, in insert: mouse Sertoli). The degree of co-localization varied between the cells, suggesting either a cell-cycle or stage-dependent behavior. Several targets of SUMO involved in transcriptional regulation and microRNA biogenesis (CDC5 $\left(F_{1}, F_{2}, G_{1}, G_{2}\right)$ and STK31 $\left(H_{1}, H_{2}\right)$ ) showed some overlap with SUMO that resulted in sparse orange signals detectable primarily in the DAPI-poor areas of spermatocytes. These areas may correspond to intrachromosomal territories (Branco \& Pombo 2006).

\section{Discussion}

In this study, IP and western blot analysis with an antiSUMO1 antibody were used to identify SUMO targets in testicular cells. Our previous study showed similar localization and western blot patterns of SUMO1 and SUMO2/3 in testicular cells and sperm. Together with an absence of defects in spermatogenesis in SUMO1knockout mice (Zhang et al. 2008), these data suggest that the functions and targets of SUMO1 and SUMO2/3 overlap in testicular cells. In other cell types, certain proteins are preferentially modified by either SUMO1 or $2 / 3$. These data suggest that the presence of specific SUMO1 and SUMO2/3 targets during spermatogenesis cannot be excluded.

Our results suggest the role of sumoylation as a major player in the regulation of transcription, stress responses, the regulation of major enzymatic pathways, nuclearcytoplasmic transport, cell-cycle control, acrosome biogenesis, and other functions in spermatogenesis. Interestingly, proteins involved in ubiquitination and sumoylation, DNA repair, and chromatin remodeling are highly sumoylated in spermatocytes, whereas cytoskeleton proteins are highly modified by SUMO in spermatids. It is possible that these proteins are more dynamically regulated specific cell types, as consistent with their functions.

Several proteins identified as SUMO targets in this study were previously found to be sumoylated in somatic cells (studies from other groups) or in human sperm (a study from our group). These proteins included RanGAP1, MDC1, KAP1, heat shock proteins, heteronucleoproteins, and several splicing factors (Mahajan et al. 1998, Vassileva \& Matunis 2004, Li et al. 2007, 2010, Matafora et al. 2009, Yin et al. 2012, Vigodner et al. 2013). Although previous studies in mammalian cells did not identify RNAP II as a SUMO target, studies in yeast identified the largest subunit of RNAP II as being
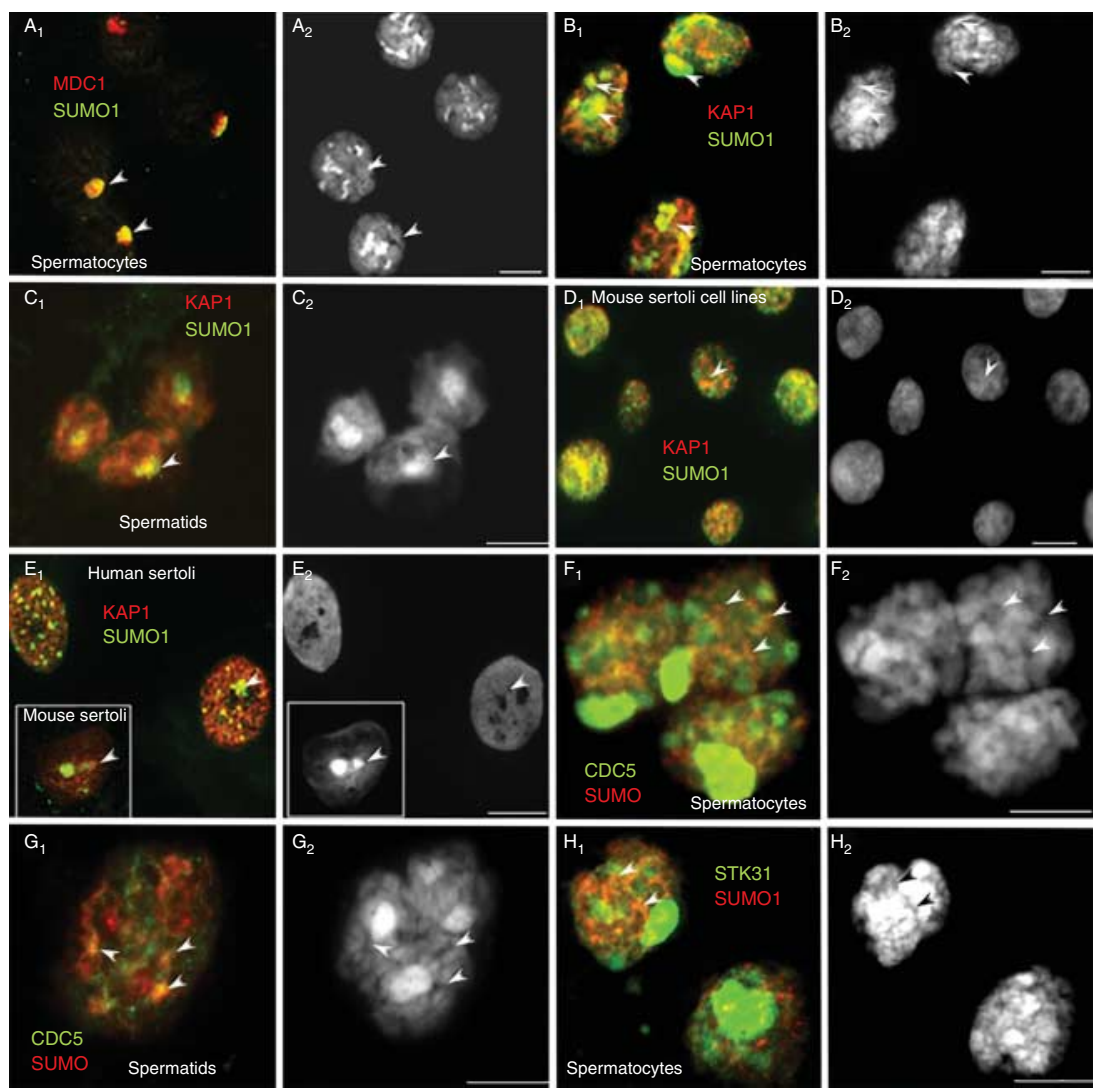
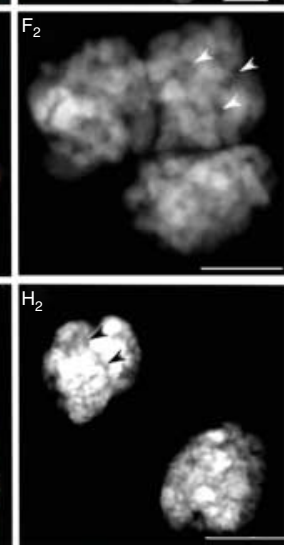

Figure 7 Immunofluorescent localization of SUMO and its putative targets in testicular cells. Targets, color-coding and cell type are indicated for each image. A mouse monoclonal antiSUMO1/GMP antibody was used in $\left(\mathrm{A}_{1}\right)$, and a rabbit monoclonal anti-SUMO1 was used in (B, C, D, E, F, G and H). Nuclei are stained by DAPI (blue). For all images, immunofluorescent staining is shown alongside the corresponding DAPI images to demonstrate the chromatin structure. Scale bar is $10 \mu \mathrm{m}$. SUMO co-localized with MDC1 in the XY body $\left(A_{1}\right.$ and $A_{2}$, arrowheads) and KAP1 in the centromeric heterochromatin and partially in the XY body of spermatocytes $\left(B_{1}\right.$ and $B_{2}$, arrowheads and arrow respectively) and chromocenters of round spermatids ( $\mathrm{C}_{1}$ and $\mathrm{C}_{2}$, arrowheads). KAP1 also co-localized with SUMO in certain areas of human and mouse Sertoli cells as well as in mouse Sertoli cell lines $\left(D_{1}, D_{2}\right.$ and $E_{1}, E_{2}$, an insert: mouse Sertoli). CDC5 $\left(F_{1}, F_{2}, G_{1}\right.$, and $\left.G_{2}\right)$, and STK31 $\left(\mathrm{H}_{1}\right.$ and $\left.\mathrm{H}_{2}\right)$ were detectable primarily in the DAPI-poor areas of cells that may correspond to intrachromasomal domains (arrowheads). 
sumoylated (Chen et al. 2009). In a similar manner, CDK1 was identified as a SUMO target in Drosophila (Nie et al. 2009). These previous data strongly support our findings and the specificity of our identification. Our results are further supported by the fact that all of the studied targets, with the exception of RNAP II and TDP-43, contain one or more consensus sumoylation sequence/s, and many of these sequences are evolutionarily conserved (Supplementary Table 2). RNAP II and TDP-43 have non-consensus sequences, but those sequences are evolutionarily conserved.

IP of endogenous proteins is challenging because sometimes only a small fraction of certain proteins can be sumoylated at a given time. In some experiments, possible sumoylated isoforms were precipitated with and anti-SUMO antibody but not with the antibody against the target protein (DDX4, Fig. 3D). These results are probably due to a lower affinity of specific antibodies to the sumoylated form of the protein. For other targets (MILI and CDC5, Figs 3C and 5C), sumoylated isoforms were only precipitated with the antibody against the target protein and not with the SUMO antibody. These results can likely be explained by a very large number of sumoylated proteins in cells and a limited ability of the anti-SUMO antibody to precipitate $100 \%$ of the sumoylated proteins, particularly those with a very low degree of sumoylation.

Localization studies support the MS results regarding a potentially diverse role of sumoylation in germ cells (Fig. 7). Several targets show a partial overlap with SUMO (orange signal). For example, KAP1 is preferentially associated with the heterochromatin structures of spermatocytes, spermatids, and Sertoli cells. Interestingly, in somatic cells KAP1 can catalyze its own sumoylation and transcriptional repression in a phosphorylation-dependent manner (Li et al. 2007, 2010). Germ cell expression of KAP1 is required for spermatogenesis in the mouse. However, the shedding of immature germ cells in mutant mice was attributed to impaired paracrine interactions between germ and Sertoli cells (Weber et al. 2002, Herzog et al. 2011). Therefore, the role and regulation of KAP1 sumoylation in the heterochromatic region of germ and Sertoli cells should be further examined.

MDC1, which co-localizes with SUMO in the $X Y$ body, was recently implicated in spreading heterochromatin over the sex chromosome during $\mathrm{MSCl}$ (Ichijima et al. 2011). In somatic cells, MDC1 is sumoylated in response to the formation of doublestranded DNA breaks (Yin et al. 2012). SUMO and several other proteins involved in heterochromatin formation were absent from the sex body of Mdc1-null spermatocytes. Based on these results, the authors of this early study concluded that MDC1 is upstream of SUMO during $\mathrm{MSCl}$. Our results complicated this interpretation somewhat because the sumoylated target itself is not present in Mdc1-null spermatocytes. MDC1 also plays a role in DNA double-stranded break repair and can be SUMO modified in response to ionizing radiation. Sumoylated MDC1 then recruits the ubiquitin ligase RNF4, which mediates ubiquitination at the DNA damage site (Yin et al. 2012). Our previous study showed that SUMO is also localized to the DNA breaks in germ cells (Shrivastava et al. 2014). These data, together with the results of this study, suggest that MDC1 can be sumoylated at the meiotic DNA breaks. Whether this modification activates ubiquitination remains to be determined, but our data support a close crosstalk between ubiquitination and sumoylation. Specifically, several ubiquitin-activating and ubiquitin-conjugating enzymes as well as ubiquitin hydrolyses were identified as SUMO targets (Table 1). Furthermore, similar to previous results from our group obtained for human sperm, ubiquitin was identified in SUMO pulldowns. These results suggest that some proteins are simultaneously modified by sumoylation and ubiquitination (Vigodner et al. 2013).

Several targets implicated in the regulation of transcription and/or microRNA biogenesis were found to interact with SUMO (Fig. 3). Interestingly, MILI was mostly sumoylated in spermatocytes. These data are consistent with a specific role for this protein during meiosis.

TDP-43 is a ubiquitously expressed transcription factor that is highly conserved through evolution (Lalmansingh et al. 2011). There has been an increasing interest in this protein since mislocalized TDP-43 was found in the intracellular ubiquitinated inclusions in the brains of patients with frontotemporal lobar degeneration with ubiquitin-positive inclusions, amyotrophic lateral sclerosis, and Alzheimer disease (Neumann et al. 2006, Lalmansingh et al. 2011). In the testis, TDP-43 regulates the spermatid-specific transcription of Acrv1, and mutations in the Acrv1 promoter-binding motifs of TDP-43 cause premature expression of Acrv1 in spermatocytes. Surprisingly, TDP-43 is also found at the Acrv1 promoter in spermatocytes, where it was suggested to regulate RNAP II pausing by an unknown mechanism. Our results revealed a striking difference in the sumoylation or SUMO-interaction of TDP-43 in spermatocytes and spermatids, suggesting that these interactions can contribute to RNAP II pausing or other cell-specific mechanisms in spermatocytes. Further studies will characterize how sumoylation regulates the functions of TDP-43 in germ cells and other tissues.

An interesting finding of this study was that several kinases (CDK1, CDC5, and STK31) were identified as being sumoylated. CDK1 (CDC2) is a crucial and indispensable regulator of both mitosis and meiosis (Diril et al. 2012). Although CDK1 was not reported to be sumoylated in somatic cells, it was identified as a target of sumoylation in the Drosophila embryo, supporting our finding (Nie et al. 2009). Further studies will need to uncover how the sumoylation of CDK1 affects mitotic and meiotic progression. 
STK31 was first identified as a germ cell-specific kinase (Wang et al. 2001) and was reported to interact with MIWI, suggesting a role in miRNA biogenesis and spermatogenesis (Bao et al. 2012). However, genetic studies utilizing mouse models have shown that STK31 is dispensable for spermatogenesis and oogenesis. Nevertheless, human STK31 was reported to be expressed in gastrointestinal cancers, including esophageal, gastric, colon, and colorectal cancers (Yokoe et al. 2008, Fok et al. 2012). The knockdown of Stk31 in colon cancer cells promotes cell differentiation and suppresses tumorigenicity (Fok et al. 2012). The role and regulation of STK31 by sumoylation in tumorigenesis remain to be characterized.

CDC5 is a DNA-binding protein involved in cell-cycle control. Similar to MILI and DDX4, CDC5 is associated with the production of microRNAs through interactions with their gene promoters and RNAP II (Zhang et al. 2013). In yeast, CDC5 is also involved in the regulation of the SUMO pathway and modulates the maintenance and dissolution of cohesion at centromeres (Baldwin et al. 2009, Attner et al. 2013). The functions of CDC5 and its regulation by post-translation modifications in mammalian spermatogenesis are not yet known.

Although the present data focused on proteins uniquely identified in the antibody fraction and not in the control fraction, some proteins, including those with an important role in spermatogenesis (such as HSP70-2, phosphorylated $\mathrm{H} 2 \mathrm{AX}$, other histones, topoisomerase 2 alpha, and PIWIL1), were identified in both fractions (data not shown). The observation that these proteins bind to beads in a non-specific manner does not exclude the possibility that sumoylation or interaction with SUMO occurs. Indeed, TOP2A was identified as a specific target of sumoylation in our previous work (Shrivastava et al. 2010). Further studies will need to be conducted to test the possible sumoylation of these proteins. Furthermore, there may be additional proteins in germ cells that are modified by sumoylation but that were below the detection level of the technique used in this study.

Interestingly, most of the sumoylated proteins analyzed in this study also non-covalently interact with SUMO or sumoylated proteins. Bioinformatics analysis revealed that only four proteins (MDC1, RNAP II, STK31, and TDP-43) contained a SIM (not shown). These data suggest that a greater number of proteins can interact with SUMO or sumoylated proteins non-covalently, regardless of the presence of a SIM.

In conclusion, this study identified and confirmed the sumoylation of several novel, previously uncharacterized SUMO targets, such as CDK1, RNAP II, CDC5, MILI, DDX4, TDP-43, and STK31. Furthermore, several proteins that were previously identified as SUMO targets in somatic cells (KAP1, MDC1) were identified as SUMO targets in germ cells. Many of these proteins have unique roles in spermatogenesis, particularly during meiotic progression. This research opens a novel avenue for further studies of SUMO at the level of individual targets. If the sumoylation sites of the selected proteins are not identified, site-directed mutagenesis can then be employed to mutate candidate lysine residues to arginine to determine whether this substitution causes the disappearance of the sumoylated isoform(s). New approaches to identify sumoylated sites with MS are under development but require further validation (Hsiao et al. 2009). After identification of the acceptor lysine residue(s), an attempt can be made to produce specific antibodies against the sumoylated form of the protein that can then be used for localization and interaction studies. Analysis of the functional consequences of the mutations in the sumoylated sites of the identified proteins, as well as other aspects of impaired sumoylation in germ cells, can be addressed both in vitro and in vivo.

\section{Supplementary data}

This is linked to the online version of the paper at http://dx.doi. org/10.1530/REP-15-0239.

\section{Declaration of interest}

The authors declare that there is no conflict of interest that could be perceived as prejudicing the impartiality of the research reported.

\section{Funding}

This study was supported by the NIH, NICHD, and Academic Research Enhancement Award 1R15HD067944-01A1 (M Vigodner). Undergraduate student research was supported by Selma and Jacques $\mathrm{H}$ Mitrani Foundation.

\section{References}

Alkuraya FS, Saadi I, Lund JJ, Turbe-Doan A, Morton CC \& Maas RL 2006 SUMO1 haploinsufficiency leads to cleft lip and palate. Science 313 1751. (doi:10.1126/science.1128406)

Andersen JS, Matic I \& Vertegaal AC 2009 Identification of SUMO target proteins by quantitative proteomics. Methods in Molecular Biology 497 19-31. (doi:10.1007/978-1-59745-566-4_2)

Attner MA, Miller MP, Ee LS, Elkin SK \& Amon A 2013 Polo kinase Cdc5 is a central regulator of meiosis I. PNAS 110 14278-14283. (doi:10.1073/ pnas.1311845110)

Baldwin ML, Julius JA, Tang X, Wang Y \& Bachant J 2009 The yeast SUMO isopeptidase Smt4/Ulp2 and the polo kinase Cdc5 act in an opposing fashion to regulate sumoylation in mitosis and cohesion at centromeres. Cell Cycle 8 3406-3419. (doi:10.4161/cc.8.20.9911)

Bao J, Wang L, Lei J, Hu Y, Liu Y, Shen H, Yan W \& Xu C 2012 STK31(TDRD8) is dynamically regulated throughout mouse spermatogenesis and interacts with MIWI protein. Histochemistry and Cell Biology 137 377-389. (doi:10.1007/s00418-011-0897-9)

Barysch SV, Dittner C, Flotho A, Becker J \& Melchior F 2014 Identification and analysis of endogenous SUMO1 and SUMO2/3 targets in mammalian cells and tissues using monoclonal antibodies. Nature Protocols 9 896-909. (doi:10.1038/nprot.2014.053)

Bayer P, Arndt A, Metzger S, Mahajan R, Melchior F, Jaenicke R \& Becker J 1998 Structure determination of the small ubiquitin-related modifier SUMO-1. Journal of Molecular Biology 280 275-286. (doi:10.1006/ jmbi.1998.1839) 
Bellve AR, Cavicchia JC, Millette CF, O'Brien DA, Bhatnagar YM \& Dym M 1977 Spermatogenic cells of the prepuberal mouse. Isolation and morphological characterization. Journal of Cell Biology 74 68-85. (doi:10.1083/jcb.74.1.68)

Blomster HA, Imanishi SY, Siimes J, Kastu J, Morrice NA, Eriksson JE \& Sistonen L 2010 In vivo identification of sumoylation sites by a signature tag and cysteine-targeted affinity purification. Journal of Biological Chemistry 285 19324-19329. (doi:10.1074/jbc.M110.106955)

Branco MR \& Pombo A 2006 Intermingling of chromosome territories in interphase suggests role in translocations and transcription-dependent associations. PLoS Biology 4 e138. (doi:10.1371/journal.pbio.0040138)

Brown PW, Hwang K, Schlegel PN \& Morris PL 2008 Small ubiquitinrelated modifier (SUMO)-1, SUMO-2/3 and SUMOylation are involved with centromeric heterochromatin of chromosomes 9 and 1 and proteins of the synaptonemal complex during meiosis in men. Human Reproduction 23 2850-2857. (doi:10.1093/humrep/den300)

Campbell M \& Izumiya Y 2012 Post-translational modifications of Kaposi's sarcoma-associated herpesvirus regulatory proteins - SUMO and KSHV. Frontiers in Microbiology 3 31. (doi:10.3389/fmicb.2012.00031)

Chen X, Ding B, LeJeune D, Ruggiero C \& Li S 2009 Rpb1 sumoylation in response to UV radiation or transcriptional impairment in yeast. PLOS ONE 4 e5267. (doi:10.1371/journal.pone.0005267)

Chupreta S, Holmstrom S, Subramanian L \& Iniguez-Lluhi JA 2005 A small conserved surface in SUMO is the critical structural determinant of its transcriptional inhibitory properties. Molecular and Cellular Biology 25 4272-4282. (doi:10.1128/MCB.25.10.4272-4282.2005)

Cramer P 2006 Recent structural studies of RNA polymerases II and III. Biochemical Society Transactions 34 1058-1061. (doi:10.1042/ BST0341058)

Diril MK, Ratnacaram CK, Padmakumar VC, Du T, Wasser M, Coppola V, Tessarollo L \& Kaldis P 2012 Cyclin-dependent kinase 1 (Cdk1) is essential for cell division and suppression of DNA re-replication but not for liver regeneration. PNAS 109 3826-3831. (doi:10.1073/pnas. 1115201109)

Fok KL, Chung CM, Yi SQ, Jiang X, Sun X, Chen H, Chen YC, Kung HF, Tao Q, Diao R et al. 2012 STK31 maintains the undifferentiated state of colon cancer cells. Carcinogenesis 33 2044-2053. (doi:10.1093/carcin/ bgs246)

Goldberg M, Stucki M, Falck J, D'Amours D, Rahman D, Pappin D, Bartek J \& Jackson SP 2003 MDC1 is required for the intra-S-phase DNA damage checkpoint. Nature 421 952-956. (doi:10.1038/nature01445)

Golebiowski F, Matic I, Tatham MH, Cole C, Yin Y, Nakamura A, Cox J, Barton GJ, Mann M \& Hay RT 2009 System-wide changes to SUMO modifications in response to heat shock. Science Signaling 2 ra24. (doi:10.1126/scisignal.2000282)

Hannoun Z, Greenhough S, Jaffray E, Hay RT \& Hay DC 2010 Post-translational modification by SUMO. Toxicology 278 288-293. (doi:10.1016/j.tox.2010.07.013)

Herzog M, Wendling O, Guillou F, Chambon P, Mark M, Losson R \& Cammas F 2011 TIF1 $\beta$ association with HP1 is essential for postgastrulation development, but not for Sertoli cell functions during spermatogenesis. Developmental Biology 350 548-558. (doi:10.1016/j. ydbio.2010.12.014)

Hsiao HH, Meulmeester E, Frank BT, Melchior F \& Urlaub H 2009 "ChopNSpice," a mass spectrometric approach that allows identification of endogenous small ubiquitin-like modifier-conjugated peptides. Molecular \& Cellular Proteomics 8 2664-2675. (doi:10.1074/mcp. M900087-MCP200)

Ichijima Y, Ichijima M, Lou Z, Nussenzweig A, Camerini-Otero RD, Chen J, Andreassen PR \& Namekawa SH 2011 MDC1 directs chromosome-wide silencing of the sex chromosomes in male germ cells. Genes and Development 25 959-971. (doi:10.1101/gad.2030811)

Kerscher O 2007 SUMO junction - what's your function? New insights through SUMO-interacting motifs EMBO Reports 8 550-555. (doi:10. 1038/sj.embor.7400980)

Kunin M, Dmitrieva NI, Gallazzini M, Shen RF, Wang G, Burg MB \& Ferraris JD 2010 Mediator of DNA damage checkpoint 1 (MDC1) contributes to high $\mathrm{NaCl}$-induced activation of the osmoprotective transcription factor TonEBP/OREBP. PLoS ONE 5 e12108. (doi:10.1371/ journal.pone.0012108)
Kuramochi-Miyagawa S, Kimura T, ljiri TW, Isobe $T$, Asada N, Fujita $Y$, Ikawa M, Iwai N, Okabe M, Deng W et al. 2004 Mili, a mammalian member of piwi family gene, is essential for spermatogenesis. Development 131 839-849. (doi:10.1242/dev.00973)

Lalmansingh AS, Urekar CJ \& Reddi PP 2011 TDP-43 is a transcriptional repressor: the testis-specific mouse acrv1 gene is a TDP-43 target in vivo. Journal of Biological Chemistry 286 10970-10982. (doi:10.1074/jbc. M110.166587)

La Salle S, Sun F, Zhang XD, Matunis MJ \& Handel MA 2008 Developmental control of sumoylation pathway proteins in mouse male germ cells. Developmental Biology 321 227-237. (doi:10.1016/j. ydbio.2008.06.020)

La Salle S, Sun F \& Handel MA 2009 Isolation and short-term culture of mouse spermatocytes for analysis of meiosis. Methods in Molecular Biology 558 279-297. (doi:10.1007/978-1-60761-103-5_17)

Lasko P 2013 The DEAD-box helicase Vasa: evidence for a multiplicity of functions in RNA processes and developmental biology. Biochimica et Biophysica Acta 1829 810-816. (doi:10.1016/j.bbagrm.2013.04.005)

Li X, Lee YK, Jeng JC, Yen Y, Schultz DC, Shih HM \& Ann DK 2007 Role for KAP1 serine 824 phosphorylation and sumoylation/desumoylation switch in regulating KAP1-mediated transcriptional repression. Journal of Biological Chemistry 282 36177-36189. (doi:10.1074/jbc. M706912200)

Li X, Lin HH, Chen H, Xu X, Shih HM \& Ann DK 2010 SUMOylation of the transcriptional co-repressor KAP1 is regulated by the serine and threonine phosphatase PP1. Science Signaling 3 ra32. (doi:10.1126/ scisignal.2000781)

Lin DY, Huang YS, Jeng JC, Kuo HY, Chang CC, Chao TT, Ho CC, Chen YC, Lin TP, Fang HI et al. 2006 Role of SUMO-interacting motif in Daxx SUMO modification, subnuclear localization, and repression of sumoylated transcription factors. Molecular Cell 24 341-354. (doi:10.1016/j.molcel.2006.10.019)

Lou Z, Minter-Dykhouse K, Franco S, Gostissa M, Rivera MA, Celeste A, Manis JP, van Deursen J, Nussenzweig A, Paull TT et al. 2006 MDC1 maintains genomic stability by participating in the amplification of ATMdependent DNA damage signals. Molecular Cell 21 187-200. (doi:10.1016/j.molcel.2005.11.025)

Luo H, Zhou Y, Li Y \& Li Q 2013 Splice variants and promoter methylation status of the Bovine Vasa Homology (Bvh) gene may be involved in bull spermatogenesis. BMC Genetics 14 58. (doi:10.1186/1471-2156-14-58)

Mahajan R, Gerace L \& Melchior F 1998 Molecular characterization of the SUMO-1 modification of RanGAP1 and its role in nuclear envelope association. Journal of Cell Biology 140 259-270. (doi:10.1083/jcb. 140.2.259)

Marchiani S, Tamburrino L, Ricci B, Nosi D, Cambi M, Piomboni P, Belmonte G, Forti G, Muratori M \& Baldi E 2014 SUMO1 in human sperm: new targets, role in motility and morphology and relationship with DNA damage. Reproduction 148 453-467. (doi:10.1530/REP14-0173)

Matafora V, D'Amato A, Mori S, Blasi F \& Bachi A 2009 Proteomics analysis of nucleolar SUMO-1 target proteins upon proteasome inhibition. Molecular \& Cellular Proteomics 8 2243-2255. (doi:10.1074/mcp. M900079-MCP200)

Matunis MJ, Coutavas E \& Blobel G 1996 A novel ubiquitin-like modification modulates the partitioning of the Ran-GTPase-activating protein RanGAP1 between the cytosol and the nuclear pore complex. Journal of Cell Biology 135 1457-1470. (doi:10.1083/jcb.135.6.1457)

Metzler-Guillemain C, Depetris D, Luciani JJ, Mignon-Ravix C, Mitchell MJ \& Mattei MG 2008 In human pachytene spermatocytes, SUMO protein is restricted to the constitutive heterochromatin. Chromosome Research 16 761-782. (doi:10.1007/s10577-008-1225-7)

Moroianu J 1998 Distinct nuclear import and export pathways mediated by members of the karyopherin $\beta$ family. Journal of Cellular Biochemistry $\mathbf{7 0}$ 231-239. (doi:10.1002/(SICl)1097-4644(19980801)70:2<231::AIDJCB9>3.0.CO;2-P)

Mukhopadhyay D \& Dasso M 2007 Modification in reverse: the SUMO proteases. Trends in Biochemical Sciences 32 286-295. (doi:10.1016/j. tibs.2007.05.002)

Myojin R, Kuwahara S, Yasaki T, Matsunaga T, Sakurai T, Kimura M, Uesugi S \& Kurihara Y 2004 Expression and functional significance of mouse paraspeckle protein 1 on spermatogenesis. Biology of Reproduction 71 926-932. (doi:10.1095/biolreprod.104.028159) 
Nacerddine K, Lehembre F, Bhaumik M, Artus J, Cohen-Tannoudji M, Babinet C, Pandolfi PP \& Dejean A 2005 The SUMO pathway is essential for nuclear integrity and chromosome segregation in mice. Developmental Cell 9 769-779. (doi:10.1016/j.devcel.2005.10.007)

Neumann M, Sampathu DM, Kwong LK, Truax AC, Micsenyi MC, Chou TT, Bruce J, Schuck T, Grossman M, Clark CM et al. 2006 Ubiquitinated TDP-43 in frontotemporal lobar degeneration and amyotrophic lateral sclerosis. Science 314 130-133. (doi:10.1126/science.1134108)

Nie M, Xie Y, Loo JA \& Courey AJ 2009 Genetic and proteomic evidence for roles of Drosophila SUMO in cell cycle control, Ras signaling, and early pattern formation. PLOS ONE 4 e5905. (doi:10.1371/journal.pone. 0005905)

Rodriguez MS, Dargemont C \& Hay RT 2001 SUMO-1 conjugation in vivo requires both a consensus modification motif and nuclear targeting. Journal of Biological Chemistry 276 12654-12659. (doi:10.1074/jbc. M009476200)

Rogers RS, Inselman A, Handel MA \& Matunis MJ 2004 SUMO modified proteins localize to the $\mathrm{XY}$ body of pachytene spermatocytes. Chromosoma 113 233-243. (doi:10.1007/s00412-004-0311-7)

Roscioli E, Di Francesco L, Bolognesi A, Giubettini M, Orlando S, Harel A, Schinina ME \& Lavia P 2012 Importin- $\beta$ negatively regulates multiple aspects of mitosis including RANGAP1 recruitment to kinetochores. Journal of Cell Biology 196 435-450. (doi:10.1083/jcb.201109104)

Sarge KD \& Park-Sarge OK 2009 Detection of proteins sumoylated in vivo and in vitro. Methods in Molecular Biology 590 265-277. (doi:10.1007/ 978-1-60327-378-7_17)

Shrivastava V, Pekar M, Grosser E, Im J \& Vigodner M 2010 SUMO proteins are involved in the stress response during spermatogenesis and are localized to DNA double-strand breaks in germ cells. Reproduction 139 999-1010. (doi:10.1530/REP-09-0492)

Shrivastava V, Marmor H, Chernyak S, Goldstein M, Feliciano M \& Vigodner M 2014 Cigarette smoke affects posttranslational modifications and inhibits capacitation-induced changes in human sperm proteins. Reproductive Toxicology 43 125-129. (doi:10.1016/j.reprotox.2013. 12.001)

Song J, Durrin LK, Wilkinson TA, Krontiris TG \& Chen Y 2004 Identification of a SUMO-binding motif that recognizes SUMO-modified proteins. PNAS 101 14373-14378. (doi:10.1073/pnas.0403498101)

Song J, Zhang Z, Hu W \& Chen Y 2005 Small ubiquitin-like modifier (SUMO) recognition of a SUMO binding motif: a reversal of the bound orientation. Journal of Biological Chemistry 280 40122-40129. (doi:10.1074/jbc.M507059200)

Stark GR \& Taylor WR 2006 Control of the G2/M transition. Molecular Biotechnology 32 227-248. (doi:10.1385/MB:32:3:227)

Stopka T, Zakova D, Fuchs O, Kubrova O, Blafkova J, Jelinek J, Necas E \& Zivny J 2000 Chromatin remodeling gene SMARCA5 is dysregulated in primitive hematopoietic cells of acute leukemia. Leukemia $\mathbf{1 4}$ 1247-1252. (doi:10.1038/sj.leu.2401807)

Suzuki T, Ichiyama A, Saitoh H, Kawakami T, Omata $\mathrm{M}$, Chung $\mathbf{C H}$, Kimura M, Shimbara N \& Tanaka K 1999 A new 30-kDa ubiquitinrelated SUMO-1 hydrolase from bovine brain. Journal of Biological Chemistry 274 31131-31134. (doi:10.1074/jbc.274.44.31131)

Tanaka SS, Toyooka Y, Akasu R, Katoh-Fukui Y, Nakahara Y, Suzuki R, Yokoyama M \& Noce T 2000 The mouse homolog of Drosophila Vasa is required for the development of male germ cells. Genes and Development 14 841-853.

Tatham MH, Rodriguez MS, Xirodimas DP \& Hay RT 2009 Detection of protein SUMOylation in vivo. Nature Protocols 4 1363-1371. (doi:10.1038/nprot.2009.128)

Vassileva MT \& Matunis MJ 2004 SUMO modification of heterogeneous nuclear ribonucleoproteins. Molecular and Cellular Biology 24 3623-3632. (doi:10.1128/MCB.24.9.3623-3632.2004)

Vigodner M 2009 Sumoylation precedes accumulation of phosphorylated $\mathrm{H} 2 \mathrm{AX}$ on sex chromosomes during their meiotic inactivation. Chromosome Research 17 37-45. (doi:10.1007/s10577-008-9006-x)

Vigodner M \& Morris PL 2005 Testicular expression of small ubiquitinrelated modifier-1 (SUMO-1) supports multiple roles in spermatogenesis: silencing of sex chromosomes in spermatocytes, spermatid microtubule nucleation, and nuclear reshaping. Developmental Biology 282 480-492. (doi:10.1016/j.ydbio.2005.03.034)
Vigodner M, Ishikawa T, Schlegel PN \& Morris PL 2006 SUMO-1, human male germ cell development, and the androgen receptor in the testis of men with normal and abnormal spermatogenesis. American Journal of Physiology. Endocrinology and Metabolism 290 E1022-E1033. (doi:10.1152/ajpendo.00527.2005)

Vigodner M, Shrivastava V, Gutstein LE, Schneider J, Nieves E, Goldstein M, Feliciano M \& Callaway M 2013 Localization and identification of sumoylated proteins in human sperm: excessive sumoylation is a marker of defective spermatozoa. Human Reproduction 28 210-223. (doi:10.1093/humrep/des317)

Vourekas A, Zheng Q, Alexiou P, Maragkakis M, Kirino Y, Gregory BD \& Mourelatos Z 2012 Mili and Miwi target RNA repertoire reveals piRNA biogenesis and function of Miwi in spermiogenesis. Nature Structural \& Molecular Biology 19 773-781. (doi:10.1038/nsmb.2347)

Wang Y \& Dasso M 2009 SUMOylation and deSUMOylation at a glance. Journal of Cell Science 122 4249-4252. (doi:10.1242/jcs.050542)

Wang PJ, McCarrey JR, Yang F \& Page DC 2001 An abundance of X-linked genes expressed in spermatogonia. Nature Genetics 27 422-426. (doi:10.1038/86927)

Wang J, Gu H, Lin H \& Chi T 2012 Essential roles of the chromatin remodeling factor BRG1 in spermatogenesis in mice. Biology of Reproduction 86 186. (doi:10.1095/biolreprod.111.097097)

Wang J, Dixon SE, Ting LM, Liu TK, Jeffers V, Croken MM, Calloway M, Cannella D, Hakimi MA, Kim K et al. 2014 Lysine acetyltransferase GCN5b interacts with AP2 factors and is required for Toxoplasma gondii proliferation. PLoS Pathogens 10 e1003830. (doi:10.1371/journal.ppat. 1003830)

Weber P, Cammas F, Gerard C, Metzger D, Chambon P, Losson R \& Mark M 2002 Germ cell expression of the transcriptional co-repressor TIF $1 \beta$ is required for the maintenance of spermatogenesis in the mouse. Development 129 2329-2337.

Wilkinson KA \& Henley JM 2010 Mechanisms, regulation and consequences of protein SUMOylation. Biochemical Journal 428 133-145. (doi:10.1042/BJ20100158)

Xiao Y, Pollack D, Nieves E, Winchell A, Callaway M \& Vigodner M 2015 Can your protein be sumoylated? A quick summary and important tips to study SUMO-modified proteins Analytical Biochemistry 477 95-97. (doi:10.1016/j.ab.2014.11.006)

Yeh ET 2009 SUMOylation and De-SUMOylation: wrestling with life's processes. Journal of Biological Chemistry 284 8223-8227. (doi:10. 1074/jbc.R800050200)

Yeh ET, Gong L \& Kamitani T 2000 Ubiquitin-like proteins: new wines in new bottles. Gene 248 1-14. (doi:10.1016/S0378-1119(00)00139-6)

Yin Y, Seifert A, Chua JS, Maure JF, Golebiowski F \& Hay RT 2012 SUMOtargeted ubiquitin E3 ligase RNF4 is required for the response of human cells to DNA damage. Genes and Development 26 1196-1208. (doi:10. 1101/gad.189274.112)

Yokoe T, Tanaka F, Mimori K, Inoue H, Ohmachi T, Kusunoki M \& Mori M 2008 Efficient identification of a novel cancer/testis antigen for immunotherapy using three-step microarray analysis. Cancer Research 68 1074-1082. (doi:10.1158/0008-5472.CAN-07-0964)

Zhang FP, Mikkonen L, Toppari J, Palvimo JJ, Thesleff I \& Janne OA 2008 Sumo-1 function is dispensable in normal mouse development. Molecular and Cellular Biology 28 5381-5390. (doi:10.1128/MCB. 00651-08)

Zhang S, Xie M, Ren G \& Yu B 2013 CDC5, a DNA binding protein, positively regulates posttranscriptional processing and/or transcription of primary microRNA transcripts. PNAS 110 17588-17593. (doi:10.1073/ pnas.1310644110)

Zhao Q, Xie Y, Zheng Y, Jiang S, Liu W, Mu W, Liu Z, Zhao Y, Xue Y \& Ren J 2014 GPS-SUMO: a tool for the prediction of sumoylation sites and SUMO-interaction motifs. Nucleic Acids Research 42 W325-W330. (doi:10.1093/nar/gku383)

Received 25 May 2015

First decision 29 June 2015

Revised manuscript received 16 October 2015

Accepted 23 November 2015 